\title{
Broad-spectrum antibodies against self-antigens and cytokines in RAG deficiency
}

Jolan E. Walter, ${ }^{1,2}$ Lindsey B. Rosen, ${ }^{3}$ Krisztian Csomos, ${ }^{1}$ Jacob M. Rosenberg, ${ }^{4}$ Divij Mathew, ${ }^{5}$ Marton Keszei, ${ }^{6}$ Boglarka Ujhazi, ${ }^{1,7}$ Karin Chen, ${ }^{8}$ Yu Nee Lee, ${ }^{2}$ Irit Tirosh, ${ }^{9}$ Kerry Dobbs, ${ }^{2}$ Waleed Al-Herz, ${ }^{10}$ Morton J. Cowan, ${ }^{11}$ Jennifer Puck, ${ }^{11}$ Jack J. Bleesing, ${ }^{12}$ Michael S. Grimley, ${ }^{12}$ Harry Malech, ${ }^{13}$ Suk See De Ravin, ${ }^{13}$ Andrew R. Gennery, ${ }^{14}$ Roshini S. Abraham, ${ }^{15}$ Avni Y. Joshi, ${ }^{16}$ Thomas G. Boyce, ${ }^{17}$ Manish J. Butte, ${ }^{18}$ Kari C. Nadeau, ${ }^{18}$ Imelda Balboni, ${ }^{18}$ Kathleen E. Sullivan, ${ }^{19}$ Javeed Akhter, ${ }^{20}$ Mehdi Adeli, ${ }^{21}$ Reem A. El-Feky, ${ }^{22}$ Dalia H. El-Choneimy, ${ }^{22}$ Chassan Dbaibo, ${ }^{23}$ Rima Wakim, ${ }^{23}$ Chiara Azzari, ${ }^{24}$ Paolo Palma, ${ }^{25}$ Caterina Cancrini, ${ }^{25}$ Kelly Capuder, ${ }^{2}$ Antonio Condino-Neto, ${ }^{26}$ Beatriz T. Costa-Carvalho, ${ }^{27}$ Joao Bosco Oliveira, ${ }^{28}$ Chaim Roifman, ${ }^{29}$ David Buchbinder, ${ }^{30}$ Attila Kumanovics, ${ }^{31}$ Jose Luis Franco, ${ }^{32}$ Tim Niehues, ${ }^{33}$ Catharina Schuetz, ${ }^{34}$ Taco Kuijpers, ${ }^{35}$ Christina Yee, ${ }^{2}$ Janet Chou, ${ }^{2}$ Michel J. Masaad, ${ }^{2}$ Raif Geha, ${ }^{2}$ Gulbu Uzel, ${ }^{3}$ Rebecca Gelman, ${ }^{36}$ Steven M. Holland, ${ }^{3}$ Mike Recher, ${ }^{37}$ Paul J. Utz, ${ }^{4,38}$ Sarah K. Browne, ${ }^{3}$ and Luigi D. Notarangelo $0^{2,39}$

'Pediatric Allergy and Immunology and the Center for Immunology and Inflammatory Diseases, Massachusetts Ceneral Hospital, Harvard Medical School, Boston, Massachusetts, USA. 2Division of Immunology, Boston Children's Hospital, Boston, Massachusetts, USA. ${ }^{3}$ Laboratory of Clinical Infectious Diseases, National Institute of Allergy and Infectious Diseases (NIAID), NIH, Bethesda, Maryland, USA. ${ }^{4}$ Department of Medicine, Division of Immunology and Rheumatology, Stanford University, Stanford, California, USA. Integrated Department of Immunology, University of Colorado at Denver, Integrated Department of Immunology, National Jewish Health, Denver, Colorado, USA. ${ }^{6}$ Department of Microbiology, Tumor and Cell Biology, Karolinska Institute, Stockholm, Sweden. 'Department of Biochemistry and Molecular Biology, University of Debrecen, Debrecen, Hungary. ${ }^{8}$ Division of Allergy, Immunology and Rheumatology, Department of Pediatrics, University of Utah, Salt Lake City, Utah, USA. ${ }^{9}$ Pediatric Rheumatology, Boston Children's Hospital, Boston, Massachusetts, USA. 10Pediatric Department, Faculty of Medicine, Kuwait University, Kuwait City, Kuwait. "Pediatric Allergy, Immunology and Blood and Marrow Transplant Division, UCSF Benioff Children's Hospital, San Francisco, California, USA. ${ }^{2}$ Division of Bone Marrow Transplantation and Immunodeficiency, Cincinnati Children's Hospital Medical Center, Cincinnati, Ohio, USA. ${ }^{13}$ Laboratory of Host Defenses, NIAID, NIH, Bethesda, Maryland, USA. ${ }^{14}$ Department of Pediatric Immunology and Institute of Cellular Medicine, Newcastle upon Tyne Hospitals, Newcastle upon Tyne, United Kingdom. ${ }^{15}$ Department of Laboratory Medicine and Pathology, Mayo Clinic, Rochester, Minnesota, USA. ${ }^{16}$ Division of Pediatric Allergy/Immunology, Department of Pediatric and Adolescent Medicine, and ${ }^{17}$ Division of Pediatric Infectious Diseases, Department of Pediatrics, Mayo Clinic, Rochester, Minnesota, USA. ${ }^{18}$ Department of Pediatrics, Division of Allergy, Immunology, Rheumatology, Stanford University, Stanford, California, USA. ${ }^{19} \mathrm{Alllergy}$ and Immunology, Children's Hospital of Philadelphia, Philadelphia, Pennsylvania, USA. ${ }^{20}$ effrey Modell Foundation Immunology Referral Center, Advocate Children's Hospital, Oak Lawn, Illinois, USA. ${ }^{21}$ Pediatrics Department, Weill Cornell Medical College, Hamad Medical Corporation, Doha, Qatar. ${ }^{22}$ Department of Pediatric Allergy and Immunology, Children's Hospital, Faculty of Medicine, Ain Shams University, Cairo, Egypt. ${ }^{23}$ Department of Pediatrics and Adolescent Medicine, American University of Beirut, Beirut, Lebanon. ${ }^{24}$ Anna Meyer Children's University Hospital, Florence, Italy. ${ }^{25}$ University Department of Paediatrics, IRCCS Bambino Cesù Children's Hospital, Rome, Italy. ${ }^{26}{ }^{2}$ epartment of Immunology, Institute of Biomedical Sciences, University of São Paulo, São Paulo, Brazil. . ${ }^{27}$ Federal University of São Paulo, São Paulo, Brazil. ${ }^{28}$ Instituto de Medicina Integral, Recife, Brazil. ${ }^{29} \mathrm{Hospital}$ for Sick Children, University of Toronto, Toronto, Ontario, Canada. ${ }^{30}$ Pediatric Hematology, CHOC Children's Hospital - UC Irvine, Orange, California, USA. ${ }^{31}$ Department of Pathology, University of Utah, Salt Lake City, Utah, USA. ${ }^{32}$ Croup of Primary Immunodeficiencies, Department of Microbiology and Parasitology, School of Medicine, University of Antioquia, Medellin, Colombia. ${ }^{33}$ Centre for Child Health and Adolescence, Helios Klinikum Krefeld Academic Hospital, Heinrich Heine University of Düsseldorf, Düsseldorf, Germany. ${ }^{34}$ Department of Pediatrics and Adolescent Medicine, University Hospital Ulm, Ulm, Germany. ${ }^{35} \mathrm{Emma}$ Children's Hospital, Academic Medical Center, Amsterdam, Netherlands. ${ }^{36}$ Dana Farber Cancer Institute, Department of Biostatistics and Computational Biology, Boston, Massachusetts, USA. ${ }^{37}$ Immunodeficiency Clinic, Medical Outpatient Unit and Immunodeficiency Lab, Department of Biomedicine, University Hospital, Basel, Switzerland. ${ }^{38}$ Institute for Immunity, Transplantation and Infection, Stanford University, Stanford, California, USA. ${ }^{39}$ Harvard Stem Cell Institute, Harvard University, Boston, Massachusetts, USA.

Patients with mutations of the recombination-activating genes (RAG) present with diverse clinical phenotypes, including severe combined immune deficiency (SCID), autoimmunity, and inflammation. However, the incidence and extent of immune dysregulation in RAG-dependent immunodeficiency have not been studied in detail. Here, we have demonstrated that patients with hypomorphic RAG mutations, especially those with delayed-onset combined immune deficiency and granulomatous/ autoimmune manifestations (CID-C/AI), produce a broad spectrum of autoantibodies. Neutralizing anti-IFN- $\alpha$ or anti-IFN- $\omega$ antibodies were present at detectable levels in patients with CID-G/AI who had a history of severe viral infections. As this autoantibody profile is not observed in a wide range of other primary immunodeficiencies, we hypothesized that recurrent or chronic viral infections may precipitate or aggravate immune dysregulation in RAG-deficient hosts. We repeatedly challenged $\operatorname{Rag} 1^{1723 C / 5723 C}$ mice, which serve as a model of leaky SCID, with agonists of the virus-recognizing receptors TLR3/MDA5, TLR7/-8, and TLR9 and found that this treatment elicits autoantibody production. Altogether, our data demonstrate that immune dysregulation is an integral aspect of RAG-associated immunodeficiency and indicate that environmental triggers may modulate the phenotypic expression of autoimmune manifestations.

Authorship note: Jolan E. Walter and Lindsey B. Rosen are co-first authors. Mike Recher and Paul J. Utz contributed equally to this work.

Conflict of interest: The authors have declared that no conflict of interest exists. Submitted: December 12, 2014; Accepted: September 3, 2015.

Reference information: J Clin Invest. 2015;125(11):4135-4148. doi:10.1172/JCI80477.

\section{Introduction}

Historically, primary immunodeficiencies (PIDs) were defined by increased susceptibility to infections. However, it is increasingly recognized that immune dysregulation, inflammation, and auto- 
immunity in particular are frequently observed in patients with PID (1-3). Deficiency of recombinase-activating genes 1 and 2 (RAG1/2) is a prototypic PID with a broad phenotypic spectrum including susceptibility to severe infections and immune dysregulation. The RAG1 and RAG2 proteins initiate the $\mathrm{V}(\mathrm{D}) \mathrm{J}$ recombination process, enabling expression of $\mathrm{T}$ and $\mathrm{B}$ lymphocyte antigen receptors and thereby promoting differentiation of $\mathrm{T}$ and $\mathrm{B}$ lymphocytes. Accordingly, functionally null $R A G 1 / 2$ mutations hamper the development of $\mathrm{T}$ and $\mathrm{B}$ lymphocytes, causing $\mathrm{T}^{-} \mathrm{B}^{-}$severe combined immune deficiency (SCID) (4). By contrast, hypomorphic RAG mutations that allow residual expression and function of the mutant protein, enabling partial $\mathrm{T}$ and $\mathrm{B}$ lymphocyte development, may cause a spectrum of phenotypes with prominent immune dysregulation, as observed in patients with Omenn syndrome (OS) (5), leaky SCID (LS) with a predominance of T cell receptor (TCR) $\gamma \delta^{+}$T cells $(6,7)$, and combined immunodeficiency with granulomatous disease and/ or autoimmunity (CID-G/AI) (8). In the latter group of patients, granulomatous lesions and autoimmunity may cause systemic disease or severe organ damage, which often dominates the clinical picture (8-13). Furthermore, 1 patient with CD4 T cell lymphopenia (TCL) associated with RAG mutations has been reported (14).

We and others have reported increased levels of autoantibodies in patients and in mouse models of RAG deficiency (15, 16). However, the frequency, diversity, and pathogenicity of autoantibodies in patients with RAG deficiency and the potential role of environmental factors in triggering autoimmunity in this condition remain to be elucidated.

Here, we report a comprehensive analysis of autoantibody specificities in patients with RAG deficiency and the correlation of these specificities with various clinical phenotypes. We demonstrate that patients with CID-G/AI in particular produce a broad spectrum of autoantibodies, including neutralizing antiIFN- $\alpha$ or - IFN- $\omega$ antibodies, in association with a previous history of severe viral infections. Chronic engagement of TLR3/MDA5, TLR7/-8, and TLR9 precipitated the production of autoantibodies in a mouse model of LS caused by Rag1 mutations. We propose that viral triggers are important in promoting autoantibody production in patients and mice with partially impaired RAG activity and that anti-cytokine antibodies may serve as a biomarker of an underlying primary immune dysfunction in these patients.

\section{Results}

Characteristics of RAG-deficient patients. We have studied 30 patientswithRAGdeficiency,including13patientswithCID-G/AI; 5 with LS; 7 with OS; 4 with SCID; and 1 with TCL. The clinical, immunological, and molecular features of these patients are reported in Supplemental Table 1; supplemental material available online with this article; doi:10.1172/JCI80477DS1.

Among the 13 patients with CID-G/AI (age range: $1-30$ years; median: 10 years), the majority (11 of $13,85 \%$ ) were females. All had a history of infections, including severe varicella infection, leading to significant complications (periorbital cellulitis, hepatitis, encephalitis, or disseminated disease) in 6 patients (CID patients 2, 3, 4, 6, 9, and 13) (46\%). One patient (CID-5) developed EBV-associated tonsillar lymphoma. Ten of the thirteen patients (77\%) with CID-G/AI had a history of cytopenias and/or other autoimmune manifestations (vitiligo, myasthenia gravis, vascu- litis, psoriasis). Furthermore, 7 of 13 CID-G/AI patients (54\%) had granulomas affecting various organs: skin, tongue, adenoids, spleen, lungs, and the perineal area. Most of the CID-G/AI patients (10 of $13,77 \%$ ) received replacement therapy with i.v. Igs (IVIGs). Eight patients (61.5\%) received hematopoietic cell transplantation (HCT) at ages ranging from 1.5 to 19 years. Five CID-G/AI patients $(38.5 \%)$ died at 5, 9, 10, 20, and 27 years of age, respectively. The causes of death included central line Candida sepsis (patient CID-8) (9); sepsis (patient CID-11); fatal Aspergillus pneumonia (patient CID-17) (17); graft-versus-host disease (GVHD) (patient CID-4); and accident after successful HCT (patient CID-1) (10). Two patients (CID-9 and -14) remain clinically stable on IVIGs and immune modulation at 30 and 17 years of age, respectively.

Among the 5 patients with LS (age range: 3 months to 18 years; median: 2 years), 4 had a history of autoimmunity, including cytopenias, alopecia, vitiligo, psoriasis, and Crohn disease (Supplemental Table 1). All 5 had a history of infections. In particular, 3 patients experienced chronic or severe CMV or adenovirus infection that, in 2 of these patients (LS-5 and LS-6), was associated with expansion of TCR $\gamma \delta^{+}$T cells. All patients required IVIGs, and $4(80 \%)$ received HCT.

Among the 7 patients with OS (age range: 3 weeks to 28 months; median: 4 months), 4 had a history of severe or recurrent bacterial, viral, or fungal infections (Supplemental Table 1). One patient (OS-11) experienced disseminated adenovirus infection. One patient (OS-10) developed autoimmune hemolytic anemia (AIHA). All OS patients received HCT at ages ranging from 2 to 21 months; 5 of these patients are alive, and 2 patients (OS- 2 and OS-10) died from GVHD.

Four patients had SCID, and 1 of these patients (SCID-5) had OS-like manifestations due to maternal T lymphocyte engraftment. Two of these patients presented with severe infections due to respiratory syncytial virus (RSV) and Pneumocystis jiroveci, respectively. All SCID patients received HCT between 2 weeks and 4 months of age. Three are alive, whereas 1 patient (SCID-5) died of veno-occlusive disease (VOD) secondary to busulfan toxicity (Supplemental Table 1),

The characteristics of each group of patients are summarized in Table 1. Functional analysis of the mutated RAG proteins showed that patients with CID-G/AI carried mutations that allowed higher levels of residual recombination activity than did those identified in patients with LS, OS, or SCID (Supplemental Table 1), confirming our recent observations (18).

Identification of multiple autoantibodies in RAG-deficient patients with PID. In an attempt to characterize autoantibody profiles in patients with RAG deficiency and to identify the most common autoantigens, we probed microarrays (generated by the Genomic and Microarray Core Facility at the University of Texas Southwestern Medical Center) containing a series of autoantigens involved in connective tissue diseases (Supplemental Table 2) with plasma from 22 patients with RAG deficiency (CID-G/AI, $n=8$; TCL, $n=1$; OS, $n=7$; LS, $n=2$; SCID, $n=4$ ) (Table 1 and Supplemental Table 1). All patients were tested for the presence of autoantibodies of the IgG isotype, and all but 1 (CID-9) were tested for autoantibodies of the IgM isotype. Fifteen of the twenty-two patients $(68 \%)$ were on Ig replacement therapy when the sample was obtained. 
A

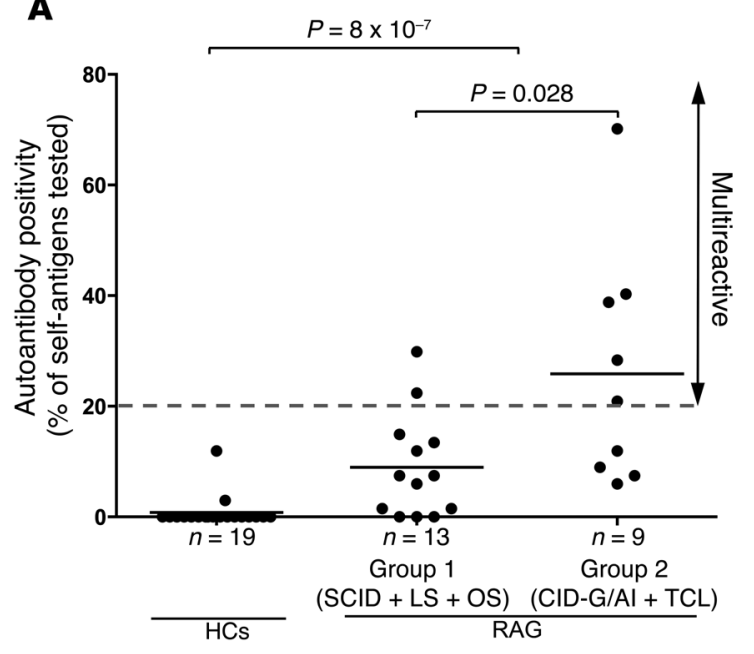

B

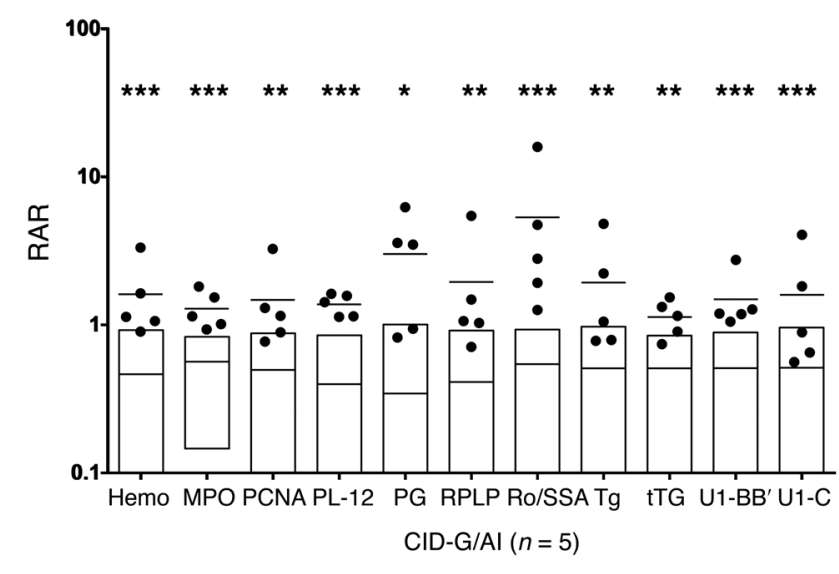

Figure 1. Autoantibodies in RAG-deficient patients as detected by protein microarray. (A) IgG autoantibodies in 19 healthy controls (HCs) and 22 patients with RAG mutations. RAG-deficient patients were divided into 2 groups according to the severity of the clinical phenotype. Group 1 included patients with T-B- SCID, OS, and LS. Group 2 included patients with delayed presentation and/or a milder phenotype: CID-C/AI and TCL. MFI was normalized to that of healthy controls (mean $+2 \mathrm{SDs}=1$ ), generating the RAR. Healthy controls had a lower percentage of positivity than did patients with RAG mutations ( $P=0.0000008)$, and group 1 had a lower percentage of positivity than did group $2(P=0.028)$ as determined by Wilcoxon test with Holm's adjustment. Samples that reacted to at least $20 \%$ of the self-antigens were defined as multireactive. (B) RAR to 11 autoantigens for which significantly higher levels of autoantibodies were found in the plasma of 5 CID-C/AI patients as compared with levels in 19 healthy controls. ${ }^{*} P<0.0005,{ }^{* *} P<0.0001$, and ${ }^{* * *} P<0.0001$ by Wilcoxon test with Holm's adjustment. Empty boxes indicate the range of RAR in healthy controls, with the bar representing the mean value. Hemo, hemocyanin; MPO, myeloperoxidase; PCNA, proliferating cell nuclear antigen; PL-12, alanyl-tRNA synthetase; PG, proteoglycan; RPLP, ribosomal phosphoprotein 0; Ro/SSA, ribonucleoprotein/Sjögren's syndrome antigen A, 52 kDa; Tg, thyroglobulin; U1-BB', U1 small nuclear ribonucleoprotein BB' 9; U1-C, U1 small nuclear ribonucleoprotein C.

In each case, individual microarrays were also probed with sera from healthy donors as negative controls ( $n=19$ and $n=16$ for IgG and IgM autoantibodies, respectively) and from a patient with systemic lupus erythematosus (SLE), who lacked RAG mutations, as a positive control. Both IgG and IgM autoantibodies were detected with increased frequency in patients with $O S$ and in those with CID-G/AI (Supplemental Figures 1 and 2, respectively). We defined a group of RAG-deficient patients as "multireactive" when their plasma contained IgG autoantibodies against at least approximately $20 \%$ (13 of 66) of the self-antigens represented on the array. The relative frequency of multireactive samples was higher in RAG-deficient patients than in healthy controls $(P=0.0000008)$ and was especially high in patients with a milder phenotype (CID-G/AI and TCL) compared with samples in patients with a more severe presentation (SCID/LS/OS) $(P=0.028)$ (Figure 1A). However, multireactive autoantibodies were also detected in 1 patient with OS (OS-5) and in the single patient with SCID and maternal engraftment (SCID-5). We identified 21 common autoantigens, defined as specific targets for IgG autoantibodies that were present in at least $20 \%$ of the patients studied (Supplemental Figure 3). Among these, 11 autoantigens were detected at significantly higher levels in multireactive patients belonging to the CID-G/AI subgroup as compared with the levels detected in healthy controls $(P<0.05)$ (Supplemental Table 3). These autoantibodies included some that are characteristic of various autoimmune diseases such as SLE, rheumatoid arthritis, and Sjögren's syndrome (Ro/SSA52Kda, U1-snRNP-BB', proteoglycan, alanyl-tRNA synthetase), and celiac disease (tissue transglutaminase [tTG]) (Figure 1B).
Distinctive anti-cytokine autoantibody signature in patients with RAG-dependent immunodeficiencies. Anti-cytokine antibodies have been detected in various PIDs and may contribute to disease phenotypes when capable of neutralizing cytokine biological activity $(19,20)$. We have previously reported on the presence of anti-IFN- $\alpha$ autoantibodies in 2 siblings with RAG deficiency who presented with autoimmunity and immunodeficiency (21). However, the frequency, specificity, and biological activity of anti-cytokine antibodies in patients with $R A G$ mutations and various clinical phenotypes have not been described. To address this issue, we probed an extended protein microarray (generated in Dr. Utz's laboratory at Stanford University) containing purified cytokines and chemokines $(n=63)$ as well as conventional autoantigens $(n=93)$ (Supplemental Table 4$)$ with plasma from 16 healthy controls and 14 RAG-deficient patients (CID-G/AI, $n=8$; TCL, $n=1$; OS, $n=3$; LS, $n=2$ ) (Table 1). A distinct panel of autoantibodies (cluster 2 ) emerged that targeted IFN- $\alpha$, IFN- $\omega$, and IL-12, mainly in the CID-G/AI, TCL, and LS subgroups (Figure 2A and Supplemental Figure 4). Significance analysis of microarrays (SAM) identified significant differences in the presence of antibodies against IFN- $\alpha$ and thyroperoxidase (TPO) in the RAG-dependent PIDs compared with that seen in controls (Figure 2B).

For further validation and characterization, a larger set of plasma samples from 23 patients with $R A G$ mutations (CID-G/AI, $n=11$; TCL, $n=1$; OS, $n=5$; LS, $n=3$; SCID, $n=3$ ) and from 15 healthy controls was tested for the presence of anti-cytokine antibodies using multiplex bead technology (Supplemental Table 5). Among the 25 distinct target cytokines tested, antibodies against IFN- $\alpha$ and IFN- $\omega$ were present in the majority of 
Table 1. Patient and sample characteristics

\begin{tabular}{|c|c|c|c|c|c|}
\hline Phenotype & CID-G/AI & TCL & LS & OS & SCID \\
\hline No. of pts. & 13 & 1 & 5 & 7 & 4 \\
\hline Age range for $\mathrm{Ab}$ testing & $1-30 \mathrm{yr}$ & $18 \mathrm{yr}$ & $5 d-13 y r$ & $5 \mathrm{wk}-2.5 \mathrm{yr}$ & $1-20$ mo \\
\hline Pts. with granulomas (\%) & $7(54 \%)$ & $0 \%$ & $0 \%$ & $0 \%$ & $0 \%$ \\
\hline Pts. with infections (\%) & $13(100 \%)$ & $1(100 \%)$ & $5(100 \%)$ & $4(57 \%)$ & $2(50 \%)$ \\
\hline Pts. with severe viral infections & $6(43 \%)$ & $1(100 \%)$ & $3(60 \%)$ & $1(14 \%)$ & $1(25 \%)$ \\
\hline Pts. treated with HCT & $8(61.5 \%)$ & $0(0 \%)$ & $4(80 \%)$ & $7(100 \%)$ & $4(100 \%)$ \\
\hline Age range of HCT-treated pts. & $1.5-19 \mathrm{yr}$ & - & $18 \mathrm{mo}-18 \mathrm{yr}$ & $2-21 \mathrm{mo}$ & 2 wk-4 mo \\
\hline Overall survival & $8(61.5 \%)$ & $1(100 \%)$ & $5(100 \%)$ & $5(71 \%)$ & $3(75 \%)$ \\
\hline Cause of death & $\begin{array}{c}\text { Sepsis (2), GVHD (1), } \\
\text { Aspergillus pneumonia (1), } \\
\text { accident (1) }\end{array}$ & - & - & GVHD (2) & $\operatorname{VOD}(1)$ \\
\hline Multiplex bead assay $(n=23)$ & $7 / 11(63.6 \%)$ & $1 / 1$ & $2 / 3(66 \%)$ & $1 / 5(20 \%)$ & $1 / 3(33 \%)$ \\
\hline ELISA $(n=18)$ & $5 / 9(56 \%)$ & $1 / 1$ & $2 / 3(66 \%)$ & $1 / 3(33 \%)$ & $0 / 2$ \\
\hline Subjects with anti-IFN- $\alpha$-neut. Abs & $6 / 6(100 \%)$ & $1 / 1$ & $2 / 2(100 \%)$ & $1 / 1(100 \%)$ & $0 / 1$ \\
\hline Subjects with anti-IFN- $\omega$-neut. Abs & $5 / 5(100 \%)$ & $1 / 1$ & $2 / 2(100 \%)$ & $0 / 1(100 \%)$ & - \\
\hline
\end{tabular}

F, female; M, male; man., manifestations; neut., neutralizing; pts., patients.

patients with CID-G/AI (7 of 11, 63.6\%), TCL (1/1), and LS (2 of 3, $66 \%)$, but were less frequently detected in patients with OS ( 1 of $5,20 \%$ ) and SCID ( 1 of $3,33.3 \%$ ). By contrast, none of the 15 healthy controls had detectable antibodies against IFN- $\alpha$ or IFN- $\omega$. In addition, antibodies targeting IL-12, IL-22, TNF- $\alpha$, IFN- $\gamma$, and IL- 6 were seen in a minority of patients (Figure $2 \mathrm{C}$ ). Of note, the anti-TNF- $\alpha$ autoantibodies detected in patients CID-12 and CID-14 likely reflected prior treatment with infliximab, a chimeric monoclonal antibody against TNF- $\alpha$. Of the 2 patients with anti-IFN- $\gamma$ antibodies, CID-12 had chronic mycobacterial infection prior to HCT. Most of the anti-cytokine antibodies detected were of the IgG1 isotype (data not shown). None of the patients had antibodies against BAFF as determined by the extended protein microarray (generated in Dr. Utz's laboratory at Stanford University) (Supplemental Table 4).

The presence of anti-IFN- $\alpha,-$ IFN- $\omega$, and -IL-12 antibodies was further validated by ELISA in a subgroup of 18 RAG-deficient patients from whom samples were available for testing (CID-G/AI, $n=9$; TCL, $n=1$; OS, $n=3$; LS, $n=3$; SCID, $n=2$; Figure $2 \mathrm{D}$ and Table 1), and the correlation with the results of autoantibody testing by multiplex bead assay is shown in Supplemental Figure 5.

To investigate whether use of IVIG may contribute to increased levels of anti-cytokine antibodies in RAG-deficient patients on Ig replacement therapy, we used ELISA to analyze anti-IFN- $\alpha$, anti-IFN- $\omega$, and anti-IL-12 antibody levels in 5 IVIG preparations diluted to a final concentration of $600 \mathrm{mg} / \mathrm{dl}$ IgG. Significantly lower levels of anti-cytokine antibodies were detected in the IVIG preparations as compared with serum levels in patients with RAG deficiency and a CID-G/AI phenotype $(n=8)$ and in 1 patient with OS (Supplemental Figure 6).

To assess whether a similar pattern of anti-cytokine antibodies may also be observed in other PIDs, we applied multiplex bead technology to study the presence of anti-cytokine antibodies in the plasma of patients with partial DiGeorge syndrome $(n=19)$, chronic granulomatous disease (CGD) $(n=7)$, and other PIDs (common variable immunodeficiency [CVID], $n=8$; polysaccharide antibody deficiency, $n=3$; ataxia-telangiectasia, $n=3)$. IgG autoantibodies targeting granulocyte CSF (G-CSF) and IFN- $\gamma$ were detected in 3 patients with partial DiGeorge syndrome, and TNF- $\alpha$ and IFN- $\gamma$ antibodies were detected in 2 patients with CGD. One patient with ataxia-telangiectasia had a combination of anti-IFN- $\alpha$ and anti-IFN- $\omega$ antibodies. In summary, patients with a PID other than RAG deficiency are also found to have anti-cytokine antibodies - but not to the degree found in patients with $R A G$ mutations - and with a different signature of antigen specificity.

Finally, we searched for IgM antibodies against various cytokines (IFN- $\alpha$, IFN- $\omega$, IL-17, and IL-22) in plasma from 4 CID-G/AI patients (CID-2,-3, -9, and -12); 2 LS patients (LS-6 and -7); and 1 OS (OS-11) patient, all of whom had tested positive for IFN- $\alpha$ and/or IFN- $\omega$ IgG antibodies. Only low levels of IgM autoantibodies were detected, comparable to what we observed in healthy controls $(n=9)$ (data not shown).

In summary, anti-cytokine antibodies were analyzed by 1 or more of 3 methods (autoantigen protein microarray, multiplex bead assay, ELISA) in plasma from 23 patients with $R A G$ mutations, 15 of whom had also been tested for conventional autoantibodies. The 
A

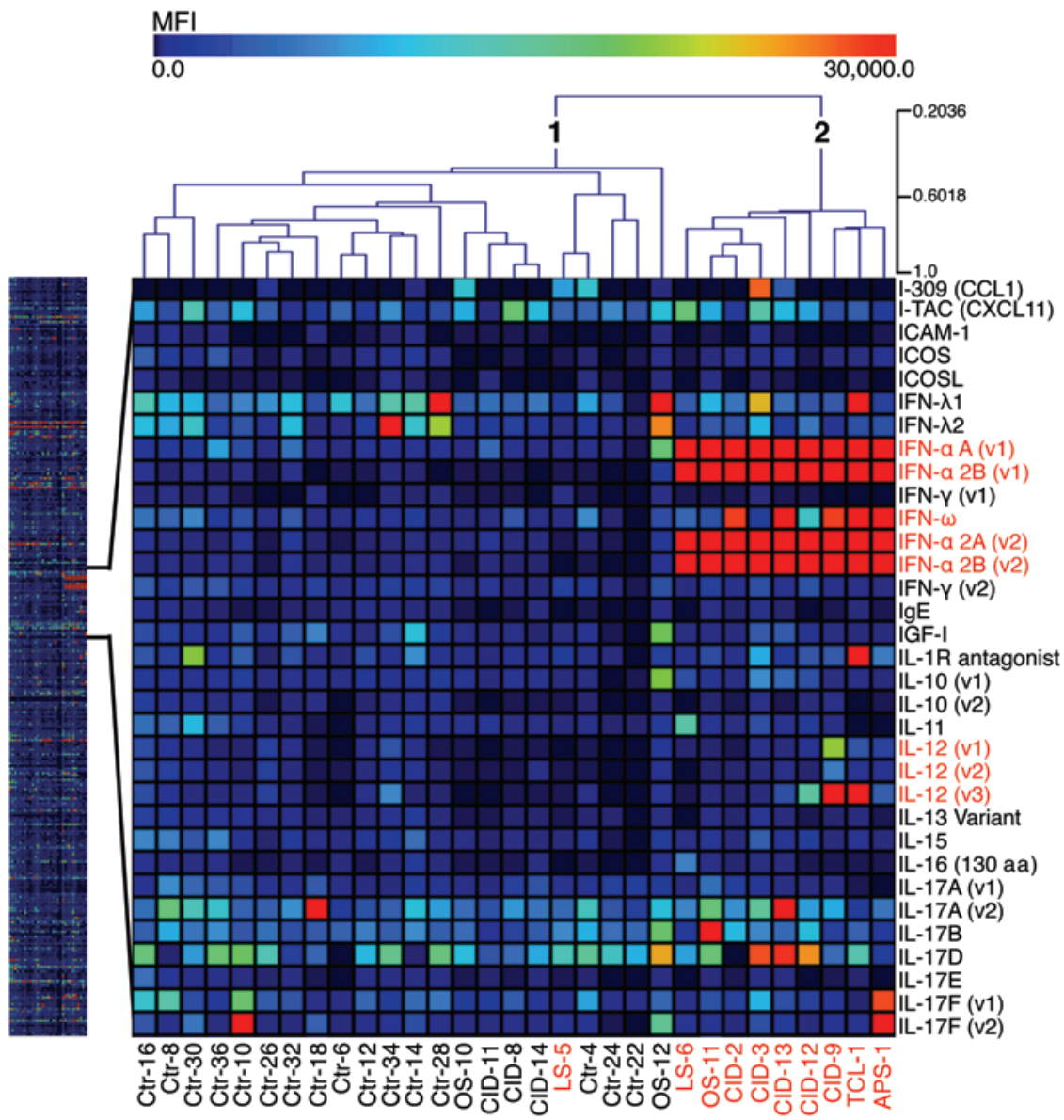

B

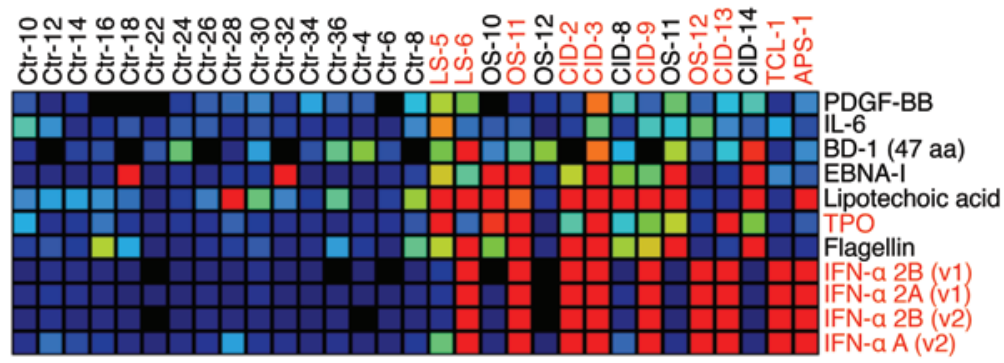

Figure 2. Anti-cytokine antibodies in RAG-deficient patients. (A) Heatmap of autoantibody reactivity. Plasma samples from 16 healthy controls (Ctr), 14 RAG-deficient patients (OS, $n=3$; LS, $n=2$; CID-G/ Al, $n=8$; idiopathic CD4+ $4^{+} \mathrm{CL}, n=1$ ), and 1 patient with APS-1 were tested for anticytokine antibodies. The complete array is shown in the left panel, and the area with the highest reactivity is magnified on the right. Antibodies against IFN- $\alpha$, IFN- $\omega$, and IL-12 (in red) were detected with high MFI in cluster 2, including RAG-deficient patients and APS-1 patients as a positive control. (B) Elevated levels of antibodies against TPO and IFN- $\alpha$ were detected in RAG-deficient patients as compared with healthy controls using SAM after 10,000 permutations of the data with an FDR of less than 0.00001. (C) Multiplex bead assay for anti-cytokine antibodies. Levels of antibodies targeting IFN- $\alpha$, IFN- $\omega$, IL-12p70, IFN- $\gamma$, IFN- $\beta$, TNF- $\alpha$, and IL-22 in healthy controls $(n=15)$ and RAG-deficient patients $(n=23)$, grouped by phenotype: SCID, $n=3$; LS, $n=3$; OS, $n=5$; CID-G/ Al, $n=11$; and TCL, $n=1$. (D) Detection of anti-IFN- $\alpha-2 \mathrm{~A},-$ IFN- $\omega$, and -IL-12p70 antibodies by ELISA. Plasma samples were assayed for IgG autoantibodies at a 200 -fold dilution. RAG-deficient patients included those with SCID $(n=2)$; LS $(n=3)$; OS ( $n=3)$; CID-C/AI $(n=9)$; and TCL $(n=1)$. In both $\mathbf{C}$ and $\mathbf{D}$, floating bars indicate the range of values for each autoantibody in healthy controls ( $n=15$ in C; $n=6$ in D).

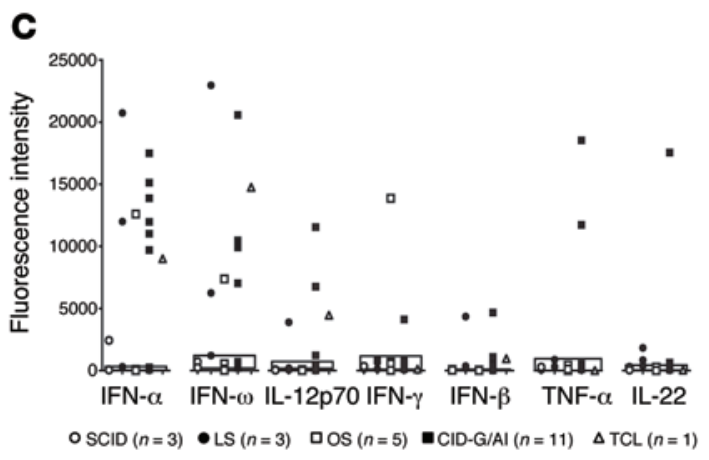

D

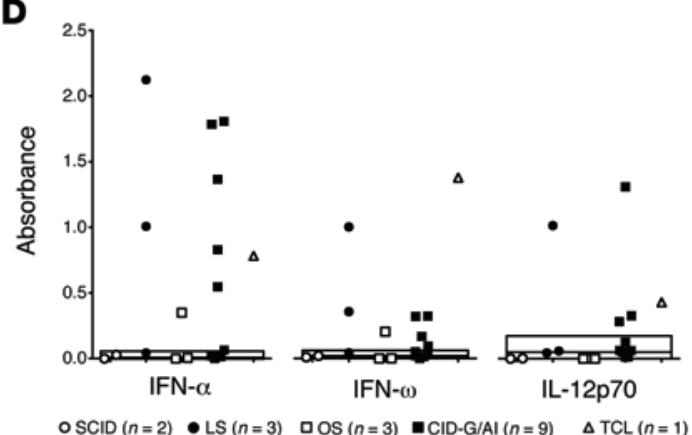

results consistently identified a distinctive signature of $\operatorname{IgG}$ anticytokine antibodies, especially in patients with CID-G/AI.

Anti-cytokine antibodies in patients with RAG-dependent immunodeficiencies have neutralizing activity. To study the possible biological role of the anti-cytokine antibodies detected, we evaluated their neutralizing activity in a subset of patients for whom we had sufficient plasma available. We assessed the ability of plasma from healthy donors or from RAG-deficient patients containing anticytokine autoantibodies to prevent either phosphorylation of the STAT molecules STAT1, -3 , and -4 upon stimulation of peripheral 
A
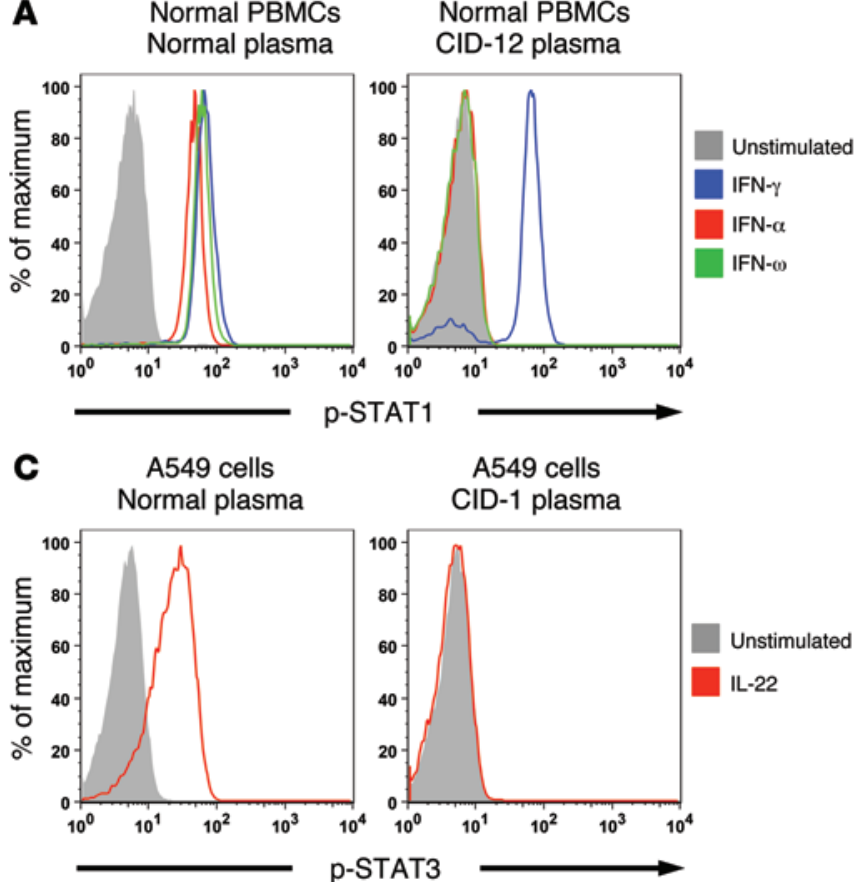

B Normal lymphoblasts Normal plasma

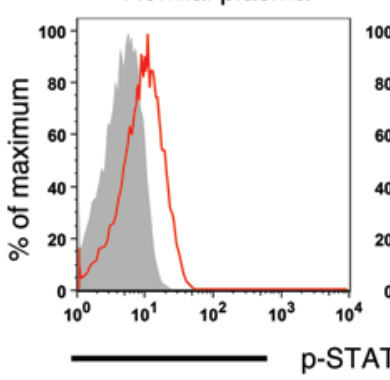

D Jurkat 3 उT 8 cells Normal plasma

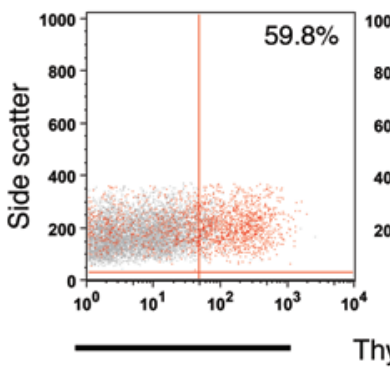

Normal lymphoblasts CID-1 plasma

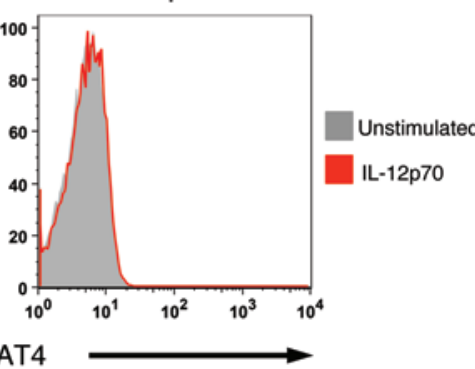

Jurkat 3 T8 cells CID-12 plasma

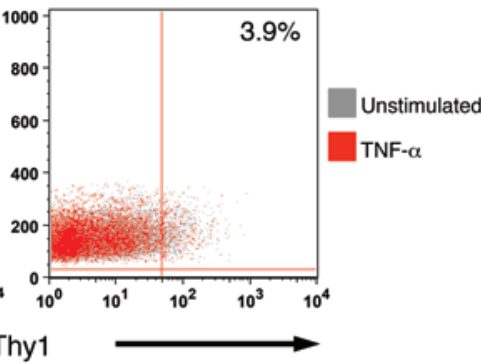

Figure 3. Neutralizing activity of anti-cytokine antibodies. (A) Neutralizing activity of anti-IFN- $\alpha$, anti-IFN- $\gamma$, and anti-IFN- $\omega$ antibodies. Normal PBMCs were incubated in the presence of plasma from a healthy control (left panel) or from a RAG-deficient patient (right panel), and left unstimulated or stimulated with either IFN- $\alpha$, IFN- $\gamma$, or IFN- $\omega$. STAT1 phosphorylation was measured by flow cytometry. The neutralizing effect of plasma from patient CID-12 on IFN- $\alpha$ (red), and IFN- $\omega$ (green), but not on IFN- $\gamma$ (blue), is shown as a representative example. (B) Neutralizing activity of anti-IL-12p70 antibodies. Normal lymphoblasts were incubated in the presence of plasma from a healthy control (left panel) or from RAG-deficient patient CID-1 as a representative example (right panel) and left unstimulated or stimulated with IL-12p70. STAT4 phosphorylation was measured by flow cytometry. (C) Neutralizing activity of anti-IL-22 autoantibodies. A549 adenocarcinomic human alveolar basal epithelial cells were incubated in the presence of plasma from a healthy control (left panel) or from RAG-deficient patient CID-1 as a representative example (right panel) and left unstimulated or stimulated with IL-22. STAT3 phosphorylation was measured by flow cytometry. (D) Neutralizing activity of anti-TNF- $\alpha$ antibodies. Jurkat 3T8 cells were incubated in the presence of plasma from a healthy control (left panel) or from RAG-deficient patient CID-12 as a representative example (right panel) and left unstimulated or stimulated with TNF- $\alpha$ overnight. Thy1 surface expression was measured by flow cytometry.

blood mononuclear cells (PBMCs) with the appropriate cytokines (IFN- $\alpha$, IFN- $\omega$, IFN- $\gamma$, IL-22, and IL-12), or induction of thymocyte antigen 1 (Thy1) surface expression in Jurkat 3T8 cells upon stimulation with TNF- $\alpha$. Data on the presence and neutralizing activity of anti-cytokine antibodies are summarized in Supplemental Table 6, and representative panels are shown in Figure 3, A-D, for each anti-cytokine antibody tested.

In particular, except for patient SCID-3 with a low titer of anti-IFN- $\alpha$ antibodies, all other plasma samples containing anti-IFN- $\alpha$ autoantibodies ( 6 of 6 CID-G/AI, 1 of 1 TCL, 2 of 2 LS, and 1 of 1 OS) inhibited IFN- $\alpha$-induced STAT1 phosphorylation (Figure 3A and Supplemental Table 6). Similarly, all but 1 of the samples containing anti-IFN- $\omega$ antibodies (5 of 5 CID-G/ AI, 1 of 1 TCL, 2 of 2 LS, and 0 of 1 OS) blocked IFN- $\omega$-induced STAT1 phosphorylation (Figure 3A and Supplemental Table 6). In 3 patients with anti-IL-12 antibodies ( 2 patients with CID-G/AI and 1 patient with TCL), plasma from patient CID-1 completely prevented IL-12-induced STAT4 phosphorylation (Figure 3B), while samples from the patient with TCL and from patient CID-9 were partially neutralizing or failed to neutralize IL-12, respectively (Supplemental Table 6). Anti-IL-22 autoantibodies were detected in 2 patients; in the case of patient CID-1, anti-IL-22 antibodies were fully neutralizing (Figure 3C), whereas patient
LS-7 had anti-IL-22 antibodies at low titers, and their neutralizing activity could not be tested. The anti-TNF- $\alpha$ antibodies detected in 2 patients with CID-G/AI were neutralizing as determined by TNF- $\alpha$-induced Thy1 surface expression in Jurkat 3T8 cells (Figure 3D). Finally, the anti-IFN- $\gamma$ antibodies detected in patients CID-12 and OS-5 did not demonstrate neutralizing activity as determined by the STAT1 phosphorylation assay (Supplemental Table 6). These results show that the anti-cytokine antibodies were antagonistic and could substantially reduce signaling via engagement of the respective cytokine receptors.

Anti-cytokine antibodies are associated with severe viral infections in patients with RAG-dependent immunodeficiencies. To investigate possible triggers of anti-IFN- $\alpha / \omega$ antibodies, we searched the medical records for relevant clinical commonalities and found that 4 of the 6 patients with CID-G/AI (patients CID-2, -3, -9, and -13) and the single patient with TCL with neutralizing anti-IFN- $\alpha / \omega$ antibodies had a history of severe varicella. Furthermore, both patients with LS and neutralizing anti-IFN- $\alpha$ antibodies (LS-6 and LS-7) had histories of CMV infection, and 1 patient (LS-7) also experienced adenovirus infection, which had also been recorded in the single patient with OS (OS-11) with anti-IFN- $\alpha / \omega$ antibodies (Table 1 and Supplemental Table 1). Of note, in patients TCL-1 and CID-9, anti-cytokine antibodies persisted for many years 
after complicated varicella infection (Supplemental Table 1). In summary, the majority of subjects with significant levels of anti-cytokine antibodies had a history of severe viral infections. In order to determine whether severe varicella infection alone could be associated with the presence of anti-cytokine antibodies, regardless of RAG deficiency, we tested plasma samples from 5 patients with a previous history of severe varicella infection and a lack of obvious immunodeficiency $(n=1)$ or the presence of other, non-RAG-dependent, forms of PID $(n=4)$, including 2 patients with ORAI1 deficiency, 1 patient with DOCK2 deficiency, and 1 patient with SCID with an unknown genetic defect. None of these patients had detectable levels of anti-cytokine antibodies. This result suggests that a history of varicella infection alone is not sufficient to elicit anti-cytokine antibodies in patients with immunodeficiencies other than RAG deficiency.

Repeated TLR3/MDA5 stimulation increases autoantibody production in a mouse model of LS due to Rag1 mutation. The occurrence of autoimmune cytopenias has been previously reported in patients with LS due to RAG mutations and CMV infection $(6,7)$, and our new data confirm that autoantibodies (including anti-cytokine antibodies) are more commonly detected in patients with $R A G$ mutations with a history of viral infections. Previous work in mice has shown that high-dose administration of poly(I:C), a ligand for TLR3/MDA5 that mimics viral infection, results in increased innate immune responses in multiple models of immune deficiency (22). Furthermore, TLR3/MDA5, TLR7/-8, or TLR9 engagement converts T lymphocyte autoreactivity into overt autoimmune disease in a $\mathrm{T}$ lymphocyte-dependent murine model of diabetes (23). On the basis of these observations, we tested the hypothesis that repeated stimulation of TLR viral sensors may trigger autoantibody production in a mouse model with hypomorphic Rag mutations. We injected low doses of TLR3, TLR7/-8, and TLR9 agonists (or PBS as a negative control) i.p. weekly for 12 weeks into 6- to 8-week-old mice with a homozygous Rag1 S723C mutation ( $\mathrm{mut} / \mathrm{mut}$ mice, a model of LS) $(15,24)$ and their wild-type (WT) littermates. After the 12-week period of weekly injections, the mice were tested for the presence of autoantibodies in the plasma using a microarray similar to that used to analyze the presence of autoantibodies in patients. As shown in Figure 4A, a subset of $\mathrm{mut} / \mathrm{mut}$ mice (8 of $15,53.3 \%$ ) produced autoantibodies even at baseline, confirming previous data of immune dysregulation in this model of LS $(15,16)$. Within 12 weeks after the initiation of weekly injections of TLR3/MDA5, TLR7/-8, and TLR9 agonists, an increase in the frequency and spectrum of IgM and IgG autoantibodies produced was observed in $\mathrm{mut} / \mathrm{mut}$ mice compared with WT mice (Figure 4, A and B). A trend toward higher levels of autoantibodies against various antigens, including single-stranded DNA (ssDNA), double-stranded DNA (dsDNA), chromatin, histone, and Ro/SSA, was observed in poly(I:C)-treated $\mathrm{mut} / \mathrm{mut}$ mice as demonstrated by higher signal intensity (Supplemental Figure 7, A and B). In comparison, autoantibody polyreactivity was not observed at baseline or after treatment with PBS in WT mice. Some autoantibody production was observed upon injection of CpG or R848 into WT mice, but only 1 of 5 (20\%) sera samples from poly(I:C)-treated WT mice showed polyreactivity (Figure 4B). Increased levels of dsDNA and ssDNA autoantibodies in mut/mut mice at baseline, and even more so after repeated poly(I:C) injection, were confirmed by ELISA (Figure 4C and Supplemental Figure 8). The kinetics of anti-ssDNA and -dsDNA autoantibody production in $\mathrm{mut} / \mathrm{mut}$ mice at intermediate time points (weeks 4 and 8) after initiation of TLR/MDA5 agonist injection is shown in Supplemental Figure 9. Anti-cytokine antibodies targeting IFN- $\alpha$, IFN- $\omega$, or IL-12 were not detected by ELISA in any of the mice, either spontaneously or after injection of TLR agonists (data not shown). In summary, these results demonstrate that repeated triggering of innate viral sensors (and TLR3/MDA5 in particular) elicits and/or increases multiple autoantibody levels in Rag-mutated mice but not in controls.

\section{Discussion}

The present report, to the best of our knowledge, involves the largest cohort of patients with the CID-G/AI phenotype and RAG deficiency described to date. Consistent with our recent observations (18), this group of patients carried mutations that allowed higher residual recombination activity when compared with other RAGdeficient patients, suggesting that the functional activity of the mutant RAG protein may affect the clinical phenotype. Interestingly, there was a marked predominance (85\%) of female individuals among the patients with CID-G/AI, whereas no sex predominance was observed in patients with LS, OS, or SCID phenotypes. Four additional patients with RAG deficiency and the CID-G/AI phenotype have been reported recently, 3 of whom were males $(12,25,26)$. Even if these cases were included, a female preponderance (70\%) would nonetheless be observed among patients with CID-G/AI. A predominance of female patients has been reported in several forms of autoimmune disease in the general population (27-31). Therefore, it is possible that besides the nature of the mutation, sex may also affect the risk of developing CID-G/AI in patients with RAG deficiency. Although patients with the CID-G/AI phenotype often survive to adulthood, even without HCT, a high proportion of the patients in our cohort eventually died due to sudden infections or transplant complications, with a low overall survival rate of $60 \%$.

Using 3 different platforms, we have demonstrated that patients with RAG deficiency, and especially those with CID-G/AI, produce a broad spectrum of autoantibodies. Increased autoantibody production in patients with hypomorphic $R A G$ mutations may reflect impairment of both central and peripheral $B$ cell tolerance $(15,16)$. In particular, reexpression of the RAG proteins during B cell development is essential to trigger further $\mathrm{V}(\mathrm{D}) \mathrm{J}$ recombination and receptor editing, thus reducing the proportion of immature B cells with self-reactive specificity that are released from the bone marrow (BM) (32-34). This mechanism is compromised in patients and mice with $R A G$ mutations $(15,16)$. Furthermore, impairment of B cell development in RAG deficiency is associated with increased serum levels of BAFF, which may promote survival of self-reactive immature B cells $(15,16)$. Interestingly, none of the patients included in this study showed evidence of anti-BAFF antibodies, which represented a cardinal feature in a recent study of patients with SLE (35).

We have demonstrated that patients with hypomorphic $R A G$ mutations have a distinctive signature of anti-cytokine antibodies, particularly those against IFN- $\alpha$, IFN- $\omega$, and IL- 12 . This profile 
$\mathbf{A}$

\begin{tabular}{llc} 
WTWT & NZMt/mut & MRL \\
\hline
\end{tabular}

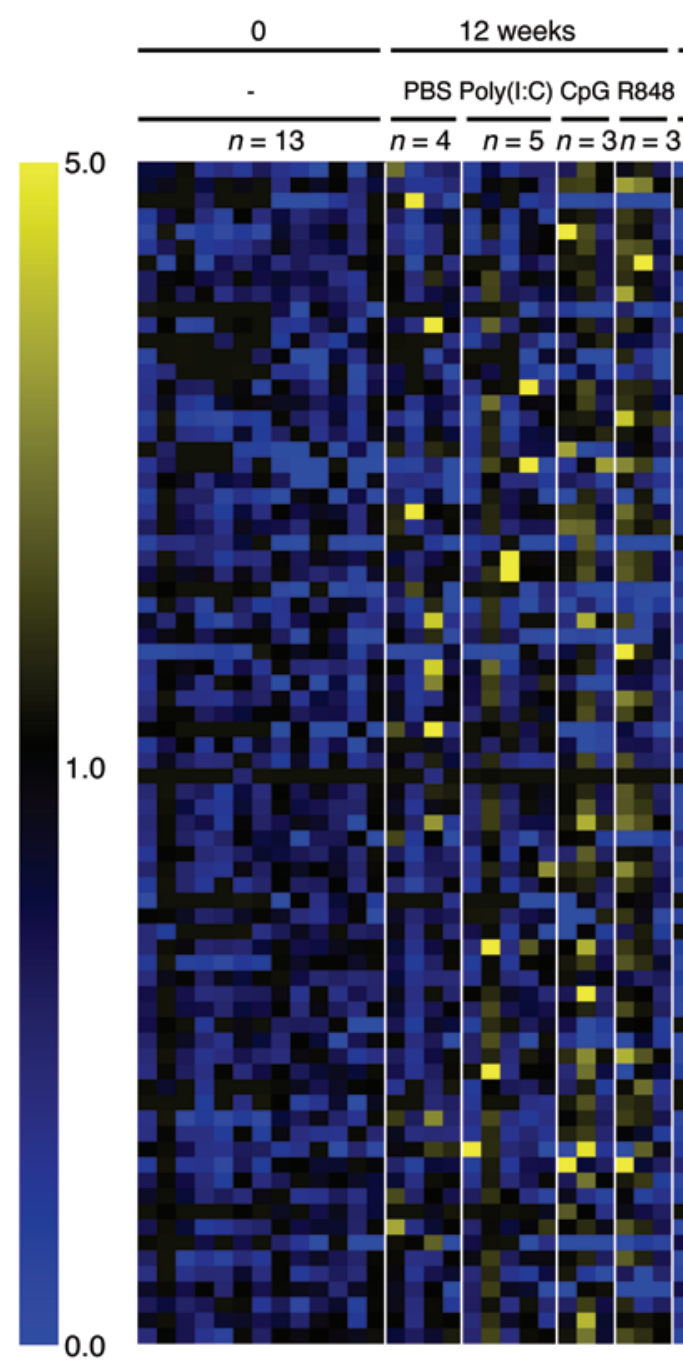

0 12 weeks

$\frac{-}{n=15} \frac{\mathrm{PBS}}{n=4} \frac{\mathrm{Poly}(\mathrm{l}: \mathrm{C})}{n=8} \frac{\mathrm{CpG}}{n=9} \frac{\mathrm{R} 848}{n=8}{ }_{n=5}$

B

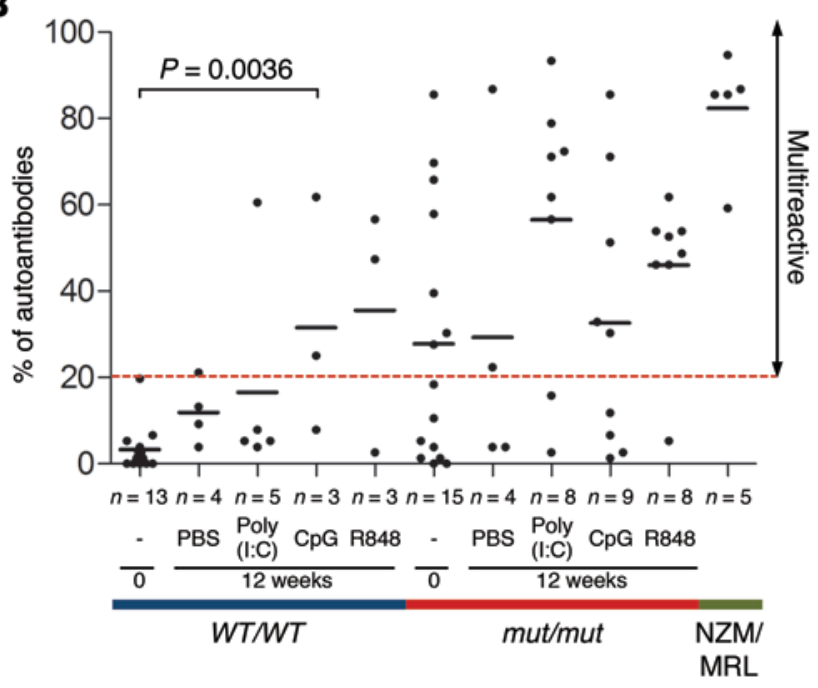

C

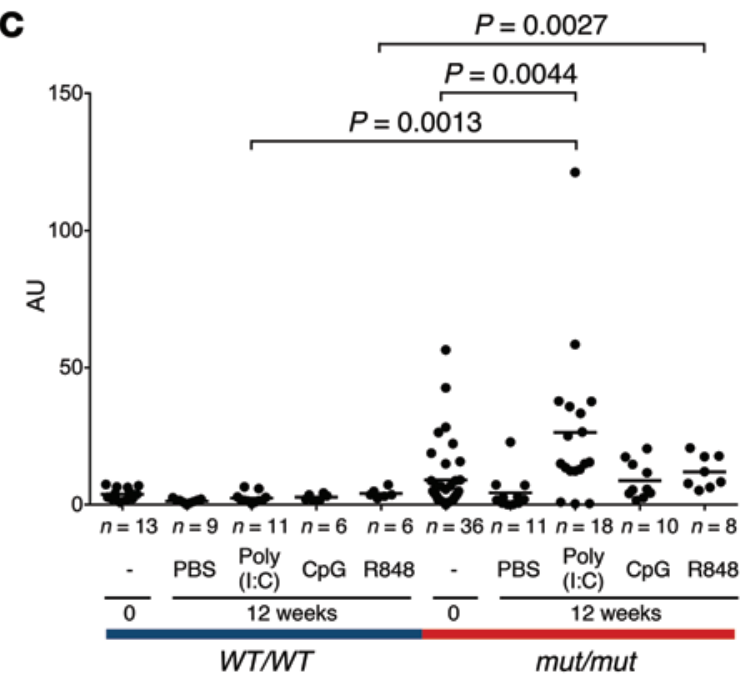


Figure 4. Autoantibody reactivity in Rag15723c/5723C (mut/mut) mice after stimulation with TLR3/MDA5, TLR7/-8, and TLR9 agonists. (A) Detection of IgG autoantibodies by protein microarray. Six- to eight-week-old mut/mut and WT/WT mice received a weekly i.p. injection of either PBS, low-dose poly(I:C), R848, or CpG. The presence of IgG antibodies against self-antigens in plasma samples was determined at day 0 and week 12 of treatment. Plasma pooled from lupus-prone NZM/MRL mice served as a positive control. Scale bars represent the MFI fold increase (blue to yellow) of autoantibody reactivity as compared with the mean $+2 \mathrm{SDs}$ of the MFI in samples from WT/WT mice. (B) Frequency of autoantibodies in mut/mut mice after TLR/MDA5 stimulation. Multireactive samples were defined as containing autoantibodies against greater than or equal to $20 \%$ of 76 self-antigens. Wilcoxon test with Holm's correction was used to compare results in WT/WT and mut/mut mice at baseline (week 0 ) and for each of the treatments and approached significance at week $O(P=0.0101)$. When comparing each of the 4 treatments with week 0 separately in WT/WT and in mut/mut mice, only CpC in WT/WT mice was significantly different $(P=0.0036)$. (C) Validation of dsDNA antibodies by ELISA. Plasma samples were diluted 200-fold. Wilcoxon test with Holm's correction was used to compare results in WT/WT and mut/mut mice at baseline (week 0 ) and for each of the treatments as well as to compare each of the 4 treatments with week 0 separately in WT/WT and mut/mut mice. Results from 3 separate experiments were pooled.

has been confirmed by 3 independent platforms (microarray, multiplex bead assay, and ELISA) and has not been identified in patients with other forms of PID analyzed herein. On the other hand, anti-IFN- $\alpha$, anti-IFN- $\omega$, and anti-IL-12 antibodies have been previously reported in patients with autoimmune polyglandular syndrome type 1 (APS-1) $(19,36)$ and thymoma $(19,36,37)$. APS-1 is a monogenic autoimmune disorder caused by mutations of the gene encoding the autoimmune regulator (AIRE), a transcription factor that is expressed by mature medullary thymic epithelial cells (mTECs) and allows expression of tissue-specific peptides that are presented by thymic epithelial cells and DCs to developing thymocytes, thereby permitting negative selection of self-reactive T cells (38). Impaired expression of AIRE has been also reported in the thymus of patients with thymoma (39-41). Interestingly, we have previously demonstrated profound abnormalities of thymic architecture, mTEC differentiation, and AIRE expression in patients with RAG deficiency $(42,43)$. The identification of a similar anti-cytokine antibody signature in patients with primary (APS-1) and secondary (thymoma and RAG mutations) deficiencies of thymic AIRE expression strongly suggests that impairment of central $\mathrm{T}$ cell tolerance plays a critical role in promoting the production of a restricted pattern of anti-cytokine antibodies in these conditions. On the other hand, unlike patients with APS-1, RAG-deficient patients did not develop anti-IL-17 antibodies. Consistent with this, candidiasis was not a common feature in the RAG-deficient patients described here, whereas it is a cardinal feature of APS-1 (18).

Circulating anti-IFN- $\alpha$ antibodies have been reported in autoimmune conditions, in SLE in particular (44-47), and even in healthy individuals, albeit at low frequency $(37,48-50)$. Furthermore, pharmaceutical Ig preparations may contain variable amounts of neutralizing anti-cytokine antibodies, including those targeting IFN- $\alpha$ (51). While most of the RAG-deficient patients studied were on Ig replacement therapy, significantly lower levels of anti-IFN- $\alpha$, anti-IFN- $\omega$, and anti-IL-12 antibodies were detected in various lots of IVIG preparations as compared with those found in serum from RAG-deficient patients $(P=0.0004$, $P=0.00067$, and $P=0.0043$, respectively) (Supplemental Figure 6). Moreover, anti-cytokine antibodies were also detected in 2 patients with RAG mutations who were not on IVIG replacement therapy. Finally, no anti-cytokine antibodies were detected in the serum of 8 patients with CVID, all of whom were on IVIG therapy.

Taken together, our data indicate that hypomorphic $R A G$ mutations are associated with an increased risk of producing a characteristic profile of anti-cytokine antibodies, particularly those against IFN- $\alpha$ and IFN- $\omega$.
While $\mathrm{B}$ and $\mathrm{T}$ cell tolerance defects are directly related to RAG deficiency, environmental factors may also play an important role in modifying the disease phenotype and aggravating or precipitating autoimmunity in patients with hypomorphic $R A G$ mutations. In particular, we have identified a clear association between the presence of neutralizing anti-IFN- $\alpha$, anti-IFN- $\omega$, and anti-IL-12 antibodies and a history of severe viral infections, such as those caused by varicella zoster virus (VZV), CMV, and adenovirus. Of note, these anti-cytokine antibodies were produced in patients at a young age following severe viral infection and tended to persist for several years.

Activation of innate viral sensors may play an important role in modifying the disease phenotype in RAG-deficient hosts. Enhanced production of proinflammatory cytokines has been previously reported when PBMCs from a patient with CID-G/AI were activated in vitro with phytohemagglutinin (PHA) and poly(I:C) (10). The observation that repeated injections of TLR3/MDA5, TLR7/-8, and TLR9 agonists in mice promotes the production of a broad range of autoantibodies is consistent with the hypothesis that persistent viral infections and sustained induction of proinflammatory responses may trigger or aggravate autoimmunity in genetically susceptible hosts. Chronic elevations of IFN- $\alpha$ levels have been implicated in the development of autoimmune disease in humans, including type I diabetes, scleroderma, and lupus (52). Furthermore, anti-IFN- $\alpha$ antibodies have also been demonstrated in patients with malignancies who received IFN- $\alpha$ treatment (53), in patients with myasthenia gravis or thymoma $(36,54,55)$, and in an otherwise healthy subject with dermatomal varicella zoster reactivation that progressed to disseminated infection (56).

The neutralizing nature of most of the anti-IFN- $\alpha$ and antiIFN- $\omega$ antibodies detected in patients with CID-G/AI suggests that these cytokine-specific autoantibodies could have important biological and clinical relevance. In particular, we propose that viral infections induce a highly inflammatory milieu with increased production of IFN- $\alpha$ and IFN- $\omega$ in the absence of proper adaptive regulatory mechanisms. In the setting of impaired tolerance, neutralizing autoantibodies are generated, which may in some cases counteract elevated IFN levels. These neutralizing autoantibodies may dampen the inflammatory response but also further compromise antiviral immune responses, predisposing to infection-related complications.

Treatment of mut/mut mice with various TLR agonists enhanced the production of autoantibodies against a wide range of self-antigens, and this effect was especially prominent for poly(I:C), which is both a TLR3 and MDA5 agonist and triggers IFN- $\alpha$ production in all tissues. However, injection of 
TLR and MDA5 agonists into mut/mut mice failed to induce the anti-cytokine antibody signature observed in patients with $R A G$ mutations. It should be noted that $\mathrm{mut} / \mathrm{mut}$ mice carry a rather severe Rag1 mutation and represent a model of LS, and not of CID-G/AI. Because the anti-cytokine antibody signature was predominantly observed in patients with CID-G/AI, it is possible that development of an anti-cytokine antibody signature in vivo requires higher residual recombination activity of the mutant RAG protein in order to sustain the generation of a sufficient number of $\mathrm{T}$ and $\mathrm{B}$ lymphocytes. To formally test this hypothesis, new mouse models are needed that have Rag mutations corresponding to those identified in patients with CID-G/AI and that are capable of supporting higher levels of recombination activity. In conclusion, we have characterized the autoantibody signature of patients with RAG deficiency and various clinical phenotypes and confirmed that immune dysregulation is a cardinal feature of this condition. A distinct profile of neutralizing anti-cytokine antibodies has been identified in patients with CID-G/AI and a history of severe viral infections and may represent a novel biomarker of this condition. Chronic stimulation of viral sensors with TLR3/MDA5 agonists enhanced autoimmunity in a mouse model of RAG deficiency, supporting the notion that environmental factors, besides RAGdependent defects of central and peripheral tolerance, are involved in determining the degree of immune dysregulation associated with RAG deficiency.

\section{Methods}

Patients. Plasma samples were obtained at diagnosis, before treatment with HCT, from patients with molecularly confirmed RAG-dependent immunodeficiency and from age-matched controls. Plasma samples from patients with RAG-nondependent PIDs (partial DiGeorge syndrome, idiopathic TCL, ataxia-telangiectasia, CVID) were also obtained.

SCID, LS, and OS were defined according to criteria from the Primary Immune Deficiency Treatment Consortium (PIDTC) (57). The CID-G/AI phenotype was defined by a clinical history of recurrent infections and immune dysregulation (autoimmunity and/or granulomas).

Mouse strains. 129Sv Rag1 ${ }^{\text {S723C/S723C }}$ ( $\mathrm{mut} / \mathrm{mut}$ ) mice were previously described (24). 129Sv WT $\left(^{+/}\right)$mice were purchased from Charles River Laboratories. Mice were housed at the Karp Family Research Center (Boston Children's Hospital, Boston, Massachusetts, USA) under specific pathogen-free conditions.

Autoantigen protein microarrays. Human and mouse IgG and IgM autoantibodies were measured on an autoantigen proteomic array (Genomic and Microarray Core Facility, University of Texas Southwestern Medical Center, Dallas, Texas, USA) (58). Autoantibodies against a selection of self-antigens were determined, and their levels were visualized as heatmaps. Specifically, the human panel included 66 and 70 self-antigens for IgG and IgM, respectively. Seventy-six self-antigens were used for mouse autoantibody testing (Supplemental Table 2). Plasma ( $1 \mu \mathrm{l})$ from patients and controls was diluted 1:100 and added to the arrays in duplicate. In the human study, each microarray was also probed with plasma derived from healthy control subjects (at least 3 per array) and plasma samples from a patient with systemic lupus erythematous (1 per array) as a positive control.
Cy3-labeled anti-human IgG and Cy5-labeled anti-human IgM were used to detect autoantibodies. In the mouse experiments, a plasma pool with broad-spectrum autoantibody specificity was generated by mixing plasma samples obtained from aged NZM2410 and MRL/ lupus-prone (MRL/lpr) mice and used as a positive control. Cy3labeled anti-mouse IgG and Cy5-labeled anti-mouse IgM antibodies were used for the detection of autoantibodies. Tiff images were generated using a GenePix 4000 B scanner (Molecular Devices) with laser wavelengths of 532 (for Cy3) and 635 (for Cy5) and analyzed using GenePix Pro 6.0 software (Molecular Devices) to generate a GenePix result (GPR) file. Net fluorescence intensities (NFI), defined as the spot minus background fluorescence intensity obtained from duplicate spots, were averaged. A signal-to-noise ratio (SNR) was used as a quantitative measure of the ability to resolve the true signal from background noise, and an SNR equal to or greater than 3 was considered a true signal in contrast to the background signal. IgG- or IgM-positive controls across all samples were averaged, and a positive control in each sample was divided by the averaged positive control, generating a normalization factor (NF) for each sample. For normalization, each signal value was multiplied by the NF of each sample. As plasma samples were measured in multiple batches, a secondary normalization step was implemented using the negative control values represented on each array. Values from the negative control samples (at least 3 per array) were averaged for each antigen, and ratios between each sample (including negative control samples) and the average of negative controls plus 2 SDs were calculated to generate relative autoantibody reactivity (RAR). These data were visualized by Multiple Experiment Viewer (MeV), version 3 (DFCI) (59) and further analyzed for multireactivity and common autoantigens. The cutoff for production of a positive autoantibody (penetrance) against a particular self-antigen was defined as RAR equal to or higher than 1 . Multireactive patients were identified as patients producing autoantibodies against at least $20 \%$ (13 of 66) of the self-antigens represented on the microarray. Common autoantigens were determined as self-antigens against which IgG autoantibodies were produced in at least $20 \%$ of the patients with RAG deficiency.

Cytokine, chemokine, and protein autoantibody microarrays. Protein microarrays (generated in Dr. Utz's laboratory at Stanford University) were generated using a VersArray ChipWriter Pro microarrayer (BioRad) with a customized printhead and Silicon Microarray Spotting Pins (Parallel Synthesis Technologies) as previously described (60). Briefly, 341 purified biomolecules including autoantigens, cytokines, and chemokines were purchased from multiple vendors and printed in triplicate at dilutions of $200 \mu \mathrm{g} / \mathrm{ml}$ onto Nexterion E epoxysilanecoated glass slides (SCHOTT; catalog 1064016). A complete list of the molecules printed is provided in Supplemental Table 4. Arrays were first blocked in 7\% FBS (Omega Scientific Inc.; catalog FB-11) in PBS (Sigma-Aldrich; catalog P4244) plus 0.1\% Tween (Sigma-Aldrich; catalog P2287) (PBST) for 1 hour at $4^{\circ} \mathrm{C}$ rocking and then washed 3 times in PBST. Arrays were probed for 1 hour with plasma diluted 1:150 in $30 \% \mathrm{FBS}$ in $1 \% \mathrm{PBST}$, with rocking at $4^{\circ} \mathrm{C}$. Arrays were subjected to three 5-minute washes in $1 \%$ PBST. Plasma reactivity was detected using an Alexa Fluor 647-conjugated goat anti-human IgG secondary antibody (Jackson ImmunoResearch Laboratories Inc.; catalog 109605-044) diluted to $2.5 \mu \mathrm{g} / \mathrm{ml}$ in $30 \%$ FCS in $1 \%$ PBST for 45 minutes. Arrays were washed 3 times in PBST and dried under negative pressure. Arrays were scanned using an Agilent microarray scanner and 
bioinformatically processed using GenePix Pro 6 software (Molecular Devices). For a selected group of patients with a documented presence of IgG anti-cytokine antibodies, we also examined the level of serum IgM antibodies relative to multiple anti-cytokine antibodies (IFN- $\alpha$, IFN- $\omega$, IL-22, and IL-17) by using the above-mentioned array, except that plasma reactivity was detected using a Cy3-conjugated goat anti-human IgM secondary antibody (Jackson ImmunoResearch Laboratories Inc.; catalog 109-165-003) diluted to $1 \mu \mathrm{g} / \mathrm{ml}$ in 30\% FCS in $1 \%$ PBST for 45 minutes.

To analyze the cytokine microarray study results, mean fluorescence intensities (MFIs) were calculated across multiple replicates, and from these values were subtracted the MFI of the corresponding features probed with a secondary antibody alone to obtain final MFI values. Unsupervised hierarchical clustering of final MFI values was performed using Pearson's correlation with average linkage clustering using the $\mathrm{MeV}$ program (59). SAM was performed as previously described (61).

Anti-cytokine autoantibody determination by multiplex bead assay. Plasma samples were screened for 23 anti-cytokine antibodies using a particle-based approach. Briefly, differentially fluorescing magnetic beads (Bio-Rad; catalogs MC1-0026-01, MC1-0027-01, MC1-002801, MC1-0029-01, MC1-0034-01, MC1-0035-01, MC1-0036-01, MC1-0037-01, MC1-0043-01, MC1-0044-01, MC1-0045-01, MC1-0046-01, MC1-0052-01, MC1-0053-01, MC1-0054-01, MC10055-01, MC1-0062-01, MC1-0063-01, MC1-0064-01, and MC10065-01) were covalently coupled to $2.5 \mu \mathrm{g}$ recombinant human IFN- $\alpha$ (PBL Biomedical Laboratories; catalog 11101-2); IFN- $\beta$ (PeproTech; catalog 300-02BC); IFN- $\gamma$ (R\&D Systems; catalog 285-IF-100/ $\mathrm{CF}$ ); IFN- $\lambda 1$ (eBioscience; catalog 34-8299); IFN- $\lambda 2$ (eBioscience; catalog 34-8289); IFN- $\lambda 3$ (R\&D Systems; catalog 5259-IL-025/CF); IFN- $\omega$ (PeproTech; catalog 300-02J); IL-1 $\alpha$ (eBioscience; catalog 34-8019); IL-4 (eBioscience; catalog 34-8049); IL-6 (eBioscience; catalog 34-8069); IL-7 (eBioscience; catalog 34-8079); IL-10 (eBioscience; catalog 34-8109); IL-12p70 (R\&D Systems; catalog 219IL-005/CF); IL-15 (eBioscience; catalog 34-8159); IL-17A (R\&D Systems; catalog 317-ILB-050); IL-17F (eBioscience; catalog 34-8479); IL-18 (R\&D Systems; catalog B003-5); IL-22 (eBioscience; catalog 34-8229); IP-10 (eBioscience; catalog 34-8967); G-CSF (eBioscience; catalog 34-8523); GM-CSF (R\&D Systems; catalog 215-GM050/CF); M-CSF (PeproTech; catalog 300-25); TNF- $\alpha$ (eBioscience; catalog 34-8329); or TNF- $\beta$ (PeproTech; catalog 300-01B). Beads were combined and incubated with plasma samples at a 1:100 dilution for 30 minutes, washed, then incubated with PE-labeled goat anti-human IgG (eBioscience; catalog 12-4998) for an additional 30 minutes before being run in a multiplex assay on the Bio-Plex X200 instrument (Bio-Rad). Ig subclasses were determined in a similar manner using biotinylated mouse anti-human IgG1, IgG2, IgG3, and IgG4 antibodies (Sigma Aldrich; catalogs B6775, B3398, B3523, and B3648, respectively) revealed by streptavidin-phycoerythrin (BioRad; catalog 171-304501).

Anti-cytokine autoantibody detection by ELISA. Ninety-six-well ELISA plates (MaxiSorp; Thermo Fisher Scientific; catalog 442404) were coated with $1 \mu \mathrm{g} / \mathrm{ml}$ recombinant human IFN- $\alpha$, (Thermo Fisher Scientific; catalog BTCYT-204); IFN- $\omega$ (Thermo Fisher Scientific; catalog BMS304); or IL-12p70 (BioLegend; catalog 573004) by incubation overnight at $4^{\circ} \mathrm{C}$. Plates were then washed in PBS-Tween $0.05 \%$, blocked by incubation with the same buffer supplemented with $2 \%$
BSA, washed, and then incubated with 1:200 dilutions of plasma samples from patients or controls for 2 hours at room temperature. Plates were thoroughly washed and then incubated with HRP-conjugated Fc-specific polyclonal goat anti-human IgG antibody (Pierce; catalog 31414) for 1 hour at room temperature. After washing, HRP substrate was added (TMB; eBioscience; catalog 00-4202-56), and the reaction was terminated with sulfuric acid (Sigma-Aldrich; catalog 38294) and OD measured at $450 \mathrm{~nm}$ in an ELISA reader. Anti-cytokine autoantibody levels in RAG-deficient patients were compared with nonautoimmune controls using the Mann-Whitney $U$ test.

To test for the presence of anti-cytokine antibodies in IVIG, 5 Ig preparations (Gammagard, lot LEIM3MAB, Baxter Healthcare; and Gamunex, lots 26NLTC1, 26NNKW3, A1GC40062, and A4GE400131, Grifols) were diluted in PBS to a final IgG concentration of $600 \mathrm{mg} / \mathrm{dl}$ and analyzed by ELISA as described above.

Analysis of neutralizing activity of anti-cytokine antibodies. Analysis of the neutralizing activity of antibodies against IFN- $\alpha$, IFN- $\omega$, and IFN- $\gamma$ was performed by assessing STAT1 phosphorylation in cells stimulated with the respective cytokines, in the presence of plasma from patients or controls (10\%) (37, 62). Briefly, PBMCs $\left(5 \times 10^{5}\right.$ cells $\left./ \mathrm{ml}\right)$ were cultured in complete media consisting of RPMI 1640, 2 mM glutamine, 20 mM HEPES ( $N$-2-hydroxyethylpiperazine- $N$-2-ethanesulfonic acid), $100 \mathrm{U} / \mathrm{ml}$ penicillin, $100 \mathrm{~g} / \mathrm{ml}$ streptomycin with plasma from normal subjects or from patients (10\%) and left unstimulated or stimulated with IFN- $\alpha(1,000 \mathrm{U} / \mathrm{ml})$, IFN- $\omega(10 \mathrm{ng} / \mathrm{ml})$, or IFN- $\gamma(1,000 \mathrm{U} / \mathrm{ml})$ for 15 minutes at $37^{\circ} \mathrm{C}$. Cells were fixed, permeabilized, and stained with phosphorylated STAT1 (p-STAT1) antibody (pY701; BD Pharmingen; catalog 612597).

To evaluate the neutralizing activity of anti-IL-12 autoantibodies, normal PBMCs were cultured for 48 hours in complete RPMI with $10 \%$ FCS and 1\% PHA, washed, and cultured for 24 hours with IL-2 (1,000 U/ml, eBioscience; catalog 34-8029), then deprived of serum overnight to generate IL-12-expressing lymphoblasts. The blasts were washed and resuspended in complete RPMI with plasma from normal subjects or patients (10\%) and either left unstimulated or stimulated with IL-12 (100 ng/ml) or IFN- $\alpha(1,000 \mathrm{U} / \mathrm{ml})$ for 15 minutes. Cells were fixed and permeabilized as described above and incubated with anti-p-STAT4 antibody (pY693; BD Pharmingen; catalog 558137) at $4^{\circ} \mathrm{C}$ for 30 minutes.

Analysis of the neutralizing activity of anti-IL-22 antibodies was performed using A549 cells $\left(5 \times 10^{5} / \mathrm{ml}\right)$ cultured in complete RPMI with plasma from normal subjects or patients (10\%) that was left unstimulated or stimulated with IL-22 (100 ng/ml) for $30 \mathrm{~min}$ utes at $37^{\circ} \mathrm{C}$. Cells were fixed, permeabilized, and stained with antip-STAT3 (pY705; BD Pharmingen; catalog 557815) antibody. The assay was developed on the basis of observations detailed in a method reported by Liang et al. (63). Data were collected using FACSCalibur (BD Biosciences) and analyzed using FlowJo software, version 9.7. For analysis of the neutralizing activity of anti-TNF- $\alpha$ antibodies, induction of Thy1 expression was analyzed in Jurkat 3T8 cells stimulated with TNF- $\alpha$ (64). Briefly, Jurkat $3 \mathrm{~T} 8$ cells $\left(5 \times 10^{5} / \mathrm{ml}\right)$ were cultured in RPMI 1640 medium containing 20 mM HEPES, $100 \mathrm{U} / \mathrm{ml}$ penicillin, $100 \mathrm{~g} / \mathrm{ml}$ streptomycin, $1 \mathrm{mM}$ sodium pyruvate, $0.1 \mathrm{mM}$ MEM nonessential amino acids solution, and $0.5 \mathrm{mg} / \mathrm{ml} \mathrm{G418} \mathrm{selec-}$ tive antibiotic in the presence of plasma from normal subjects or patients (10\%) and either stimulated with TNF- $\alpha(10 \mathrm{ng} / \mathrm{ml})$ or not (unstimulated control) overnight at $37^{\circ} \mathrm{C}$. Cells were washed and 
stained with mouse anti-rat Thy1 antibody (BD Pharmingen; catalog 561409), and data were collected using FACSCalibur (BD Biosciences) and analyzed with FlowJo software, version 9.7.

Analysis of autoantibody production in mut/mut mice upon challenge with TLR/MDA5 agonists. Six- to eight-week-old WT and Rag1 $1^{\mathrm{S} 23 \mathrm{C} / \mathrm{s} 723 \mathrm{C}}$ ( $\mathrm{mut} / \mathrm{mut}$ ) mice were injected i.p. weekly for 12 weeks with either 50 $\mu \mathrm{g}$ poly(I:C) (InvivoGen; catalog TLR-PIC); $12.5 \mu \mathrm{g}$ CpG (ODN1826; InvivoGen; catalog TLR-MODN); or $5 \mu \mathrm{g}$ R848 (InvivoGen; catalog TLRL-R848); or with PBS. Blood was collected on day zero (baseline) and after 12 weeks of weekly injections, and the presence of autoantibodies in the plasma was assessed by autoantibody microarray (as described above). Anti-ssDNA and anti-dsDNA antibodies were also analyzed by ELISA as previously described, with some modifications (65). In brief, 96-well ELISA plates (MaxiSorp; Thermo Fisher Scientific; catalog 442404) were UV irradiated and then coated with 2.5 $\mu \mathrm{g} / \mathrm{ml}$ ssDNA (Sigma-Aldrich; catalog D8899) or $2 \mu \mathrm{g} / \mathrm{ml} \mathrm{dsDNA}$ (Sigma-Aldrich; catalog D4522). Samples were incubated on plates at a 1:600 dilution. Autoantibodies were detected by ELISA, as described for the detection of anti-cytokine autoantibodies, using HRP-conjugated Fc-specific polyclonal goat anti-mouse IgG antibody (Pierce; catalog 31414). Anti-dsDNA and anti-ssDNA antibody levels were expressed as arbitrary ELISA units using a standard curve prepared with serial dilutions of an NZM/MRL plasma pool with broadspectrum autoantibody specificity generated from sera from aged NZM2410 and MRL/lpr mice. Results are expressed as the OD ratio (experimental OD/background OD).

Accession numbers. Protein microarray data generated by our Stanford University collaborators (Jacob Rosenberg and Paul J. Utz) (Figure 2A and Supplemental Figure 4) are available in the NCBI's Gene Expression Omnibus (GEO) database (GEO GSE70618) $(66,67)$. We elected to add our data generated at the Genomic and Microarray Core Facility of the University of Texas Southwestern Medical Center as supplemental data (Supplemental Table 7 for human and Supplemental Table 8 for mouse data). These data were provided as a normalized data set by the core facility.

Statistics. Two-sided tests were used for all statistical analyses. Since the various outcome measures had very different variances for the various groups of patients (and for the groups of mice that were repeatedly challenged) and since the distributions did not seem to be Gaussian, we used nonparametric methods: the exact Wilcoxon rank-sum test to compare 2 groups and the exact Kruskal-Wallis test to compare more than 2 groups; calculations of these tests were done in StatXact. $P$ values were considered significant on the basis of the Holm's adjustment for multiple comparisons (68). For K comparisons, the smallest $P$ value is compared with $0.05 / \mathrm{K}$, the next smallest $P$ value with $0.05 /(\mathrm{K}-1)$, and so on until one of the ordered $P$ values is larger than the relevant Holm's cutoff. The Holm's method keeps the overall type I error (false-positive rate) as low as that obtained by the Bonferroni method but has better power (lower false-negative rate). In Figure
4, B and C (and Supplemental Figure 8), the assays were run on specimens left over from other assays, and baseline (time $=0$ ) and week 12 (after treatment) specimens were most often not available. Therefore, we could not use longitudinal methods or paired methods, but had to use 2-group methods (which have less power and hence would be less likely to be significant than longitudinal or paired methods). GraphPad Prism 6.0 (GraphPad Software) was used for data analysis in Figure 2 and Supplemental Figure 3 and for data presentation in all figures. For the autoantibody microarray, unsupervised hierarchical clustering was performed using Pearson's correlation with average linkage clustering in the $\mathrm{MeV}$ program(59). SAM was performed as previously described, with significantly different reactivities defined by an FDR of less than $0.1 \%$ after 10,000 permutations of the data (61).

Study approval. Human studies were approved by the IRBs of Children's Hospital Boston (protocol 04-09-113R) and Massachusetts General Hospital (protocol 2011-P002918). Samples from patients at all collaborating sites were obtained after written informed consent was provided by the participants or their guardians under local IRBapproved protocols and shared as deidentified specimens for secondary use. Plasma samples from patients with RAG-nondependent PIDs (partial DiGeorge syndrome, idiopathic TCL, ataxia-telangiectasia, CVID) were obtained according to protocols approved by the IRBs of Stanford University and Children's Hospital of Philadelphia and according to Declaration of Helsinki principles.

Animal studies were approved by the IACUC of Boston Children's Hospital (protocol 07-10-1445).

\section{Acknowledgments}

This work was partly supported by grants from the NIAID, $\mathrm{NIH}$ (5R01AI100887 and U54AI082973, to L.D. Notarangelo; 5K08AI103035, to J.E. Walter; and T32GM007365, to P.J. Utz); the Manton Foundation (to L.D. Notarangelo); and the Jeffrey Modell Foundation (to L.D. Notarangelo). This research was also supported in part by the Intramural Research Program of the NIAID, NIH (to L.B. Rosen, S.M. Holland, and S.K. Browne). We acknowledge Quan-Zhen Li and Jinchun Zhou of the Genomics and Microarray Core Facility at the University of Texas Southwestern Medical Center for autoantibody profiling using autoantigen microarrays.

Address correspondence to: Luigi D. Notarangelo, Division of Immunology, Boston Children's Hospital, Karp Research Building, Room 10217, One Blackfan Circle, 10th floor, Room 10217, Boston, Massachusetts 02115, USA. Phone: 617.919.2277; E-mail: Luigi.Notarangelo@childrens.harvard.edu. Or to: Jolan E. Walter, Division of Allergy Immunology, Massachusetts General Hospital for Children, 275 Cambridge Street, Suite 530, Boston, Massachusetts 02114, USA. Phone: 617.726.8707; E-mail: jewalter@mgh.harvard.edu.

\footnotetext{
1. Carneiro-Sampaio M, Coutinho A. Tolerance and autoimmunity: lessons at the bedside of primary immunodeficiencies. Adv Immunol. 2007;95:51-82.

2. Liston A, Enders A, Siggs OM. Unravelling the association of partial T-cell immunodeficiency and immune dysregulation. Nat Rev Immunol. 2008;8(7):545-558.
}

3. Pessach I, Walter J, Notarangelo LD. Recent advances in primary immunodeficiencies: identification of novel genetic defects and unanticipated phenotypes. Pediatr Res. 2009;65(5 pt 2):3R-12R.

4. Schwarz K, et al. RAG mutations in human B cell-negative SCID. Science. 1996;274(5284):97-99.
5. Villa A, et al. Partial V(D)J recombination activity leads to Omenn syndrome. Cell. 1998;93(5):885-896.

6. Ehl S, et al. A variant of SCID with specific immune responses and predominance of $\gamma \Delta \mathrm{T}$ cells. J Clin Invest. 2005;115(11):3140-3148.

7. de Villartay JP, et al. A novel immunodefi- 
ciency associated with hypomorphic RAG1 mutations and CMV infection. JClin Invest. 2005;115(11):3291-3299.

8. Schuetz C, et al. An immunodeficiency disease with RAG mutations and granulomas. $N$ Engl J Med.2008;358(19):2030-2038.

9. Avila EM, et al. Highly variable clinical phenotypes of hypomorphic RAG1 mutations. Pediatrics. 2010;126(5):e1248-e1252.

10. De Ravin SS, et al. Hypomorphic Rag mutations can cause destructive midline granulomatous disease. Blood. 2010;116(8):1263-1271.

11. Henderson LA, et al. Expanding the spectrum of recombination-activating gene 1 deficiency: a family with early-onset autoimmunity. J Allergy Clin Immunol. 2013;132(4):969-971.

12. Schuetz C, et al. Lesson from hypomorphic recombination-activating gene (RAG) mutations: why asymptomatic siblings should also be tested. JAllergy Clin Immunol. 2014;133(4):1211-1215.

13. Reiff A, Bassuk AG, Church JA, Campbell E, Bing $\mathrm{X}$, Ferguson PJ. Exome sequencing reveals RAG1 mutations in a child with autoimmunity and sterile chronic multifocal osteomyelitis evolving into disseminated granulomatous disease. JClin Immunol. 2013;33(8):1289-1292.

14. Kuijpers TW, et al. Idiopathic CD4+ $\mathrm{T}$ lymphopenia without autoimmunity or granulomatous disease in the slipstream of RAG mutations. Blood. 2011;117(22):5892-5896

15. Walter JE, et al. Expansion of immunoglobulin-secreting cells and defects in B cell tolerance in Rag-dependent immunodeficiency. J Exp Med. 2010;207(7):1541-1554.

16. Cassani B, et al. Homeostatic expansion of autoreactive immunoglobulin-secreting cells in the Rag2 mouse model of Omenn syndrome. JExp Med. 2010;207(7):1525-1540.

17. Buchbinder $D$, et al. Identification of patients with RAG mutations previously diagnosed with common variable immunodeficiency disorders. J Clin Immunol. 2015;35(2):119-124.

18. Lee YN, et al. A systematic analysis of recombination activity and genotype-phenotype correlation in human recombination-activating gene 1 deficiency. J Allergy Clin Immunol. 2014;133(4):1099-1108.

19. Kisand K, et al. Chronic mucocutaneous candidiasis in APECED or thymoma patients correlates with autoimmunity to Th17-associated cytokines. JExp Med. 2010;207(2):299-308.

20. Puel A, et al. Autoantibodies against IL-17A, IL-17F, and IL-22 in patients with chronic mucocutaneous candidiasis and autoimmune polyendocrine syndrome type I. JExp Med. 2010;207(2):291-297.

21. Chen K, et al. Autoimmunity due to RAG deficiency and estimated disease incidence in RAG1/2 mutations. JAllergy Clin Immunol. 2014;133(3):880-882.

22. Kim KD, et al. Adaptive immune cells temper initial innate responses. Nat Med. 2007;13(10):1248-1252.

23. Lang KS, et al. Toll-like receptor engagement converts T-cell autoreactivity into overt autoimmune disease. Nat Med. 2005;11(2):138-145.

24. Giblin W, et al. Leaky severe combined immunodeficiency and aberrant DNA rearrangements due to a hypomorphic RAG1 mutation. Blood.
2009;113(13):2965-2975.

25. Sharapova SO, et al. Late-onset combined immune deficiency associated to skin granuloma due to heterozygous compound mutations in RAG1 gene in a 14 years old male. Hum Immunol. 2013;74(1):18-22.

26. Patiroglu T, et al. Atypical severe combined immunodeficiency caused by a novel homozygous mutation in Rag1 gene in a girl who presented with pyoderma gangrenosum: a case report and literature review. JClin Immunol. 2014;34(7):792-795.

27. Borchers AT, Naguwa SM, Shoenfeld Y, Gershwin ME. The geoepidemiology of systemic lupus erythematosus. Autoimmun Rev 2010;9(5):A277-A287.

28. Kivity S, Ehrenfeld M. Can we explain the higher prevalence of autoimmune disease in women? Exp Rev Clin Immunol. 2010;6(5):691-694.

29. Quintero OL, Amador-Patarroyo MJ, MontoyaOrtiz G, Rojas-Villarraga A, Anaya JM. Autoimmune disease and gender: plausible mechanisms for the female predominance of autoimmunity. JAutoimmun. 2012;38(2-3):J109-J119.

30. Sellner J, Kraus J, Awad A, Milo R, Hemmer B, Stuve $O$. The increasing incidence and prevalence of female multiple sclerosis - a critical analysis of potential environmental factors. Autoimmun Rev. 2011;10(8):495-502.

31. Whitacre CC. Sex differences in autoimmune disease. Nat Immunol. 2001;2(9):777-780.

32. Gay D, Saunders T, Camper S, Weigert M. Receptor editing: an approach by autoreactive B cells to escape tolerance. J Exp Med. 1993;177(4):999-1008.

33. Radic MZ, Erikson J, Litwin S, Weigert M. $B$ lymphocytes may escape tolerance by revising their antigen receptors. J Exp Med. 1993;177(4):1165-1173.

34. Tiegs SL, Russell DM, Nemazee D. Receptor editing in self-reactive bone marrow B cells. JExp Med. 1993;177(4):1009-1020.

35. Price JV, et al. Protein microarray analysis reveals BAFF-binding autoantibodies in systemic lupus erythematosus. J Clin Invest. 2013;123(12):5135-5145.

36. Meager A, et al. Anti-interferon autoantibodies in autoimmune polyendocrinopathy syndrome type 1 . PLoS Med. 2006;3(7):e289.

37. Burbelo PD, et al. Anti-cytokine autoantibodies are associated with opportunistic infection in patients with thymic neoplasia. Blood. 2010;116(23):4848-4858.

38. Anderson MS, et al. Projection of an immunological self shadow within the thymus by the aire protein. Science. 2002;298(5597):1395-1401.

39. Strobel P, et al. Deficiency of the autoimmune regulator AIRE in thymomas is insufficient to elicit autoimmune polyendocrinopathy syndrome type 1 (APS-1). J Pathol. 2007;211(5):563-571.

40. Suzuki E, Kobayashi Y, Yano M, Fujii Y. Infrequent and low AIRE expression in thymoma: difference in AIRE expression among WHO subtypes does not correlate with association of MG. Autoimmunity. 2008;41(5):377-382.

41. Cheng $\mathrm{MH}$, et al. Acquired autoimmune polyglandular syndrome, thymoma, and an AIRE defect. N Engl J Med.2010;362(8):764-766.

42. Cavadini P, et al. AIRE deficiency in thymus of
2 patients with Omenn syndrome. J Clin Invest. 2005;115(3):728-732.

43. Poliani PL, et al. Early defects in human T-cell development severely affect distribution and maturation of thymic stromal cells: possible implications for the pathophysiology of Omenn syndrome. Blood.2009;114(1):105-108.

44. Panem S, Check IJ, Henriksen D, Vilcek J. Antibodies to alpha-interferon in a patient with systemic lupus erythematosus. JImmunol. 1982;129(1):1-3.

45. Slavikova M, Schmeisser H, Kontsekova E, Mateicka F, Borecky L, Kontsek P. Incidence of autoantibodies against type I and type II interferons in a cohort of systemic lupus erythematosus patients in Slovakia. J Interferon Cytokine Res. 2003;23(3):143-147.

46. Morimoto AM, et al. Association of endogenous anti-interferon- $\alpha$ autoantibodies with decreased interferon-pathway and disease activity in patients with systemic lupus erythematosus. Arthritis Rheum. 2011;63(8):2407-2415.

47. Suit BE, Axelrod D, Moutsopoulos HM, Decker JL, Hooks JJ. Detection of anti-interferon antibodies in systemic lupus erythematosus. Clin Exp Rheumatol. 1983;1(2):133-135.

48. Browne SK, Holland SM. Anticytokine autoantibodies in infectious diseases: pathogenesis and mechanisms. Lancet Infect Dis. 2010;10(12):875-885.

49. Watanabe M, Uchida K, Nakagaki K, Trapnell BC, Nakata K. High avidity cytokine autoantibodies in health and disease: pathogenesis and mechanisms. Cytokine Growth Factor Rev. 2010;21(4):263-273.

50. Browne SK, et al. Adult-onset immunodeficiency in Thailand and Taiwan. N EnglJMed. 2012;367(8):725-734

51. Ross C, Svenson M, Hansen MB, Vejlsgaard GL, Bendtzen K. High avidity IFN-neutralizing antibodies in pharmaceutically prepared human IgG. JClin Invest. 1995;95(5):1974-1978.

52. Baechler EC, et al. Interferon-inducible gene expression signature in peripheral blood cells of patients with severe lupus. Proc Natl Acad Sci USA. 2003;100(5):2610-2615.

53. Trown PW, et al. Antibodies to human leucocyte interferons in cancer patients. Lancet. 1983;1(8316):81-84.

54. Bagnato F, et al. Neutralizing antibodies against endogenous interferon in myasthenia gravis patients. Eur Cytokine Netw. 2004;15(1):24-29.

55. Shiono $\mathrm{H}$, et al. Spontaneous production of antiIFN- $\alpha$ and anti-IL-12 autoantibodies by thymoma cells from myasthenia gravis patients suggests autoimmunization in the tumor. Int Immunol. 2003;15(8):903-913.

56. Pozzetto B, Mogensen KE, Tovey MG, Gresser I. Characteristics of autoantibodies to human interferon in a patient with varicella-zoster disease. J Infect Dis. 1984;150(5):707-713.

57. Shearer WT, et al. Establishing diagnostic criteria for severe combined immunodeficiency disease (SCID), leaky SCID, and Omenn syndrome: the Primary Immune Deficiency Treatment Consortium experience. J Allergy Clin Immunol. 2014;133(4):1092-1098.

58. Li QZ, et al. Protein array autoantibody profiles for insights into systemic lupus erythematosus 


\section{RESEARCH ARTICLE}

and incomplete lupus syndromes. Clin Exp Immunol. 2007;147(1):60-70.

59. Saeed AI, et al. TM4: a free, open-source system for microarray data management and analysis. BioTechniques. 2003;34(2):374-378.

60. Robinson WH, et al. Autoantigen microarrays for multiplex characterization of autoantibody responses. Nat Med. 2002;8(3):295-301.

61. Tusher VG, Tibshirani R, Chu G. Significance analysis of microarrays applied to the ionizing radiation response. Proc Natl Acad Sci U S A. 2001;98(9):5116-5121.
62. Patel SY, et al. Anti-IFN-gamma autoantibodies in disseminated nontuberculous mycobacterial infections. J Immunol. 2005;175(7):4769-4776.

63. Liang M, et al. Interleukin-22 inhibits bleomycininduced pulmonary fibrosis. Mediators Inflamm. 2013;2013:209179.

64. He KL, Ting AT. A2O inhibits tumor necrosis factor (TNF) alpha-induced apoptosis by disrupting recruitment of TRADD and RIP to the TNF receptor 1 complex in Jurkat T cells. Mol Cell Biol. 2002;22(17):6034-6045.

65. Zouali M, Stollar BD. A rapid ELISA for measure-
The Journal of Clinical Investigation

ment of antibodies to nucleic acid antigens using UV-treated polystyrene microplates. JImmunol Methods. 1986;90(1):105-110.

66. Edgar R, Domrachev M, Lash AE. Gene Expression Omnibus: NCBI gene expression and hybridization array data repository. Nucleic Acids Res. 2002;30(1):207-210.

67. Barrett T, et al. NCBI GEO: archive for functional genomics data sets - update. Nucleic Acids Res. 2013;41(Database issue):D991-D995.

68. Holm S. A simple sequentially rejective multiple test procedure. Scand J Stat. 1979;6(2):65-70. 